\title{
Effective Field Theory of Multi-Field Inflation a la Weinberg
}

\author{
Nima Khosravi* \\ Cosmology Group, African Institute for Mathematical Sciences, Muizenberg 7945, Cape Town, South Africa
}

\begin{abstract}
We employ the effective field theory approach for multi-field inflation which is a generalization of Weinberg's work [1]. In this method the first correction terms in addition to standard terms in the Lagrangian have been considered. These terms contain up to the fourth derivative of the fields including the scalar field and the metric. The results show the possible shapes of the interaction terms resulting eventually in non-Gaussianity in a general formalism. In addition generally the speed of sound is different but almost unity. Since in this method the adiabatic mode is not discriminated initially so we define the adiabatic as well as entropy modes for a specific two-field model. It has been shown that the non-Gaussianity of the adiabatic mode and the entropy mode are correlated in shape and amplitude. It is shown that even for speed close to unity large non-Gaussianities are possible in multi-field case. The amount of the non-Gaussianity depends on the curvature of the classical path in the phase-space in the Hubble unit such that it is large for the large curvature. In addition it is emphasized that the time derivative of adiabatic and entropy perturbations do not transform due to the shift symmetry as well as the original perturbations. Though two specific combinations of them are invariant under such a symmetry and these combinations should be employed to construct an effective field theory of multi-field inflation.
\end{abstract}

PACS numbers: $98.80 . \mathrm{Cq}$

\section{Contents}

\section{Introduction}

II. Briefly Review of Weinberg's Approach [1]

III. Effective Field Theory of Multi-Field Inflation

IV. A Specific Case: Adiabatic versus Entropy Perturbation

A. The Most General Two-Field Model

B. Adiabatic vs. Entropy Modes

1. The Amplitude of Non-Gaussianity

2. The Shape of Non-Gaussianity

C. An Example

D. Some Clarifications on Differences with Senatore and Zaldarriaga [4]

\section{Conclusions}

Acknowledgments

A. The Most General Lagrangian for Multi-Field Inflation

B. Comparison With Gordon et al. [10]

\section{References}




\section{INTRODUCTION}

It is generally believed that inflation can be a solution to the problems of standard cosmology such as the horizon, flatness and monopole problem. In addition to these achievements, inflation's predictions are compatible with the large scale structure and CMB fluctuations which is strong evidence in favour of inflation. The idea of inflation is the existence of an exponentially expanding universe at early times. But identifying a unique theoretical realization of this period is challenging. Many theoretical models are compatible with the observational data. For example, they are in agreement with adiabatic, nearly Gaussian fluctuations in the CMB fluctuations. To potentially discriminate between them more accurate observations, such as PLANCK, are needed. This fact is a starting point for a huge amount of work on studying non-Gaussianity of primordial fluctuations. In this field the effective field theory approach to inflation has been used to study the general possible interaction terms in the single field models [1-3] and in the multi-field context [4, 5].

The advantage of using effective theories can be seen in two regimes. Sometimes a full theory exists for an energy domain of interest. In this case the effective field theory may be performed to simplify calculations in a special subdomain of energy. In the second case the full theory is not known for the energy scales of interest. Here, by imposing the symmetries of the full theory one can still build an effective field theory. In this situation the most general form of the allowed theory, e.g. a general Lagrangian, is constructed; by comparison to observations unspecified coefficients can be fixed. Eventually the deduced effective theory may shed some lights on the real theory which is beyond our current understanding. In the special case of inflation in addition to the above reasons, the effective field theory approach can be used to justify the use of scalar fields as inflatons, as well as to provide a systematic classification of non-Gaussianities [6] among other properties.

In [2] the effective field theory has been developed for an inflationary single field model. In their approach the Lagrangian is determined by all spatially diffeomorphism invariant operators. Then the broken time invariance is reproduced by a scalar field which transforms in a definite form under diffeomorphism transformation. This scalar field is well-known as the Goldstone boson. It is shown that this scalar field represents the curvature perturbation in the validity regime of the effective field theory. In [4] the generalization to multi-field inflation is studied. The existence of more than one field in the early universe is not unnatural and the extra fields may have observable consequences. For example entropy modes (a property of multi-field models) can affect the curvature mode which is for example in the CMB. In [1] an alternative approach to [2] has been given for the effective field theory of single field inflation. In this approach all the possible terms containing up to the fourth derivative of a scalar field and the metric enter the effective Lagrangian. The final result is in agreement with [2], except some additional fourth ordered contributions tracing back to geometrical terms. In this approach, due to the presence of metric perturbations, it is possible to study the gravitational wave behavior which differs in the propagation of waves with different helicities.

In this work we are going to generalize Weinberg's approach [1] to multi-field models. In the following we will avoid the scalar metric perturbations by an appropriate gauge choice. However it is mentioned that for energy scales of interest the existence of them has no observable effects on non-Gaussianity [6, 7]. Note that in [1, 2, 4], the additional correction terms arise via space-time derivatives of the perturbations. However one can extend the effective field theory formalism to include correction terms corresponding to the potential terms. It is mentioned in [8] that they have no significant contribution to non-Gaussianities since they are highly restricted by the effectiveness of the inflationary era. But it is well-known that in the context of multi-field inflation the non-Gaussianity window becomes wider and maybe observable by the future data [9]. This fact also has been considered in the context of effective field theory for multi-field inflation in different aspects [5].

In the next section we briefly review the main results of [1]. In the third section we generalize the idea of [1] to illustrate the perturbations in the most general multi-field model. This section is based on the first appendix where we find the most Lagrangian for multi-field models. Then in the fourth section we concentrate on a two-field case, studying the evolution of adiabatic and entropy modes in details. In this section we will discuss on the amplitude and shape of non-Gaussianity in our model and illustrate a specific example. At the end of this section we infer to some differences between this approach and Senatore and Zaldarriaga [4]. In the second appendix we compare our results with [10] as a check. Finally we conclude in the last section.

\section{BRIEFLY REVIEW OF WEINBERG'S APPROACH [1]}

To generalize Einstein-Hilbert action in the presence of matter field it is possible to add the terms containing higher order derivatives in the Lagrangian in addition to the standard second order ones. In principle these additional terms can be important in the larger energy scales. As discussed in [1] this situation occurs naturally in the inflation era before horizon crossing. According to the observations; the Hubble parameter, $H$, and physical momentum, $k / a$, are equal (at horizon crossing) and much less than $M_{P}$ and even unification scale. But due to denominator of physical 
momentum in a period before horizon crossing the physical momentum has had larger value. As a consequence, considering the correction terms will help us to understand better the inflationary predictions.

Due to the above discussions Weinberg in [1] has studied the effects of the fourth order derivative terms in the Einstein-Hilbert Lagrangian in the presence of one scalar field. We are going to generalize this model by adding more than one scalar field which is interesting for its well-known observational consequences. Before that let us review very briefly[17] the main results of [1]. The starting point is the Einstein-Hilbert Lagrangian which includes the leading term

$$
\mathcal{L}_{0}=\sqrt{g}\left[-\frac{M_{P}^{2}}{2} R-\frac{M^{2}}{2} g^{\mu \nu} \partial_{\mu} \varphi \partial_{\nu} \varphi-M_{P}^{2} U(\varphi)\right]
$$

where dimensionless $\varphi \equiv \varphi_{c} / M$ is defined such that the kinetic term of $\varphi_{c}$ has the canonical form. Obviously $\varphi_{c}$ has dimension of mass. It is now easier to define the hierarchy of different derivative terms as an advantage of introduction of the scale $M$ explicitly [18]. The leading correction terms are satisfied general covariance and contain four derivatives. These term can be reduced to the following form

$$
\Delta \mathcal{L}=\sqrt{g} f(\varphi)\left(g^{\mu \nu} \varphi_{, \mu} \varphi, \nu\right)^{2}+\sqrt{g} h_{1}(\varphi) C^{\mu \nu \rho \sigma} C_{\mu \nu \rho \sigma}+\sqrt{g} h_{2}(\varphi) \varepsilon^{\kappa \lambda \mu \nu} C_{\kappa \lambda}{ }^{\rho \sigma} C_{\mu \nu \rho \sigma}
$$

where $f, h_{1}$ and $h_{2}$ are some dimensionless arbitrary functions which are assumed to be in order one 19] and $C_{\mu \nu \rho \sigma}$ is the Weyl tensor. It is noteworthy that the above terms are not all the generally covariant terms containing four derivatives. But all the allowed terms except the above terms are transformed to these terms by employing the equation of motion for the leading term as well as ignoring the surface terms (for details see Appendix A). For the scalar perturbations it is convenient to assume a gauge in which metric scalar perturbations vanish. In this gauge by splitting the scalar field to its background and perturbed parts as $\varphi=\bar{\varphi}+\delta \varphi$ the Lagrangian becomes

$$
\begin{aligned}
\mathcal{L} & =\sqrt{g}\left[-\frac{M^{2}}{2} g^{\mu \nu} \partial_{\mu} \varphi \partial_{\nu} \varphi-M_{P}^{2} U(\varphi)+f(\varphi)\left(g^{\mu \nu} \varphi, \mu \varphi, \nu\right)^{2}\right] \\
& =\overline{\mathcal{L}}-\frac{1}{2} a^{3}\left(M^{2}+4 f(\bar{\varphi}) \dot{\bar{\varphi}}^{2}\right) \times\left(-\dot{\delta}^{2}+a^{-2}(\vec{\nabla} \delta \varphi)^{2}\right) \\
& +4 a^{3} f(\bar{\varphi}) \dot{\bar{\varphi}}^{2}\left(\dot{\delta}^{2}+\dot{\delta} \dot{\varphi}^{3} / \dot{\bar{\varphi}}-a^{-2} \dot{\delta} \dot{\varphi}(\vec{\nabla} \delta \varphi)^{2} / \dot{\bar{\varphi}}+\frac{1}{4} \dot{\delta} \dot{\varphi}^{4} / \dot{\bar{\varphi}}^{2}-\frac{1}{2} a^{-2} \dot{\delta}^{2}(\vec{\nabla} \delta \varphi)^{2} / \dot{\bar{\varphi}}^{2}+\frac{1}{4} a^{-4}(\vec{\nabla} \delta \varphi)^{4} / \dot{\bar{\varphi}}^{2}\right)
\end{aligned}
$$

which reduces to the Lagrangian (19) in [1] with $\pi \equiv \delta \varphi / \dot{\bar{\varphi}}$ and $\dot{H}=-\dot{\bar{\varphi}}^{2}\left(M^{2}+4 f(\bar{\varphi}) \dot{\bar{\varphi}}^{2}\right) / 2 M_{P}^{2}$. This result is compatible with [2] with a minor disagreement. This disagreement is in the presence of quartic terms as well as quadratic and cubic terms. Due to the above Lagrangian obviously ignoring the correction term, i.e. setting $f(\varphi)=0$, results in a model with $c_{s}=1$, where $c_{s}$ is the speed of sound. But in the presence of the correction term the speed of sound is not one and may cause large non-Gaussianity. In addition the terms in the third line of (3) infer to the possible shapes of non-Gaussianities as well as their amplitude.

In this section we very briefly reviewed the idea of [1] for scalar perturbations in the context of effective field theory for inflation. In addition to scalar perturbation in [1] the tensor perturbations have been considered. It is concluded in [1] that the propagation of gravitational wave depends on the helicity of the wave in this model. In the next section we are going to generalize the above idea for a multi-field theory of inflation without considering the tensor perturbations. Since existence of multi-scalar-field has no effect on the tensor perturbations and consequently gravitational wave. The detailed calculations for the next section is in appendix $\mathrm{A}$ which is also useful for clarifying the case of one field studying very briefly in this section.

\section{EFFECTIVE FIELD THEORY OF MULTI-FIELD INFLATION}

The corresponding Lagrangian to (3) for multi-field inflation can be written as follow, which has been deduced in details in the appendix $\mathrm{A}$,

$$
\begin{aligned}
\mathcal{L}=\sqrt{g} & \left\{b_{3}^{I J K L}(\vec{\varphi}) \nabla_{\mu} \varphi_{I} \nabla^{\mu} \varphi_{J} \nabla_{\nu} \varphi_{K} \nabla^{\nu} \varphi_{L}-\frac{M^{2}}{2} \delta^{I J} \nabla_{\mu} \varphi_{I} \nabla^{\mu} \varphi_{J}-M_{P}^{2} U(\vec{\varphi})\right. \\
& \left.+a_{1}(\vec{\varphi}) R_{\mu \nu \rho \sigma} R^{\mu \nu \rho \sigma}+a_{2}(\vec{\varphi}) R_{\mu \nu} R^{\mu \nu}-\frac{M_{P}^{2}}{2} R\right\}
\end{aligned}
$$


which exactly reduces to (3) for one field case 20]. Now, by splitting the scalar fields to their background and perturbed parts $\varphi_{I}=\bar{\varphi}_{I}+\delta \varphi$ we are going to study the Lagrangian for the perturbations as well as the background. To do this we start with (4) without worrying about the tensor perturbations. The above Lagrangian can be written as $\mathcal{L}=\mathcal{L}_{0}+\Delta \mathcal{L}$ such that

$$
\begin{aligned}
& a^{-3} \mathcal{L}_{0}=b_{3}^{I J K L}(\bar{\varphi}) \dot{\bar{\varphi}}_{I} \dot{\bar{\varphi}}_{J} \dot{\bar{\varphi}}_{K} \dot{\bar{\varphi}}_{L}+\frac{M^{2}}{2} \delta^{I J} \dot{\bar{\varphi}}_{I} \dot{\bar{\varphi}}_{J}-M_{P}^{2} U(\bar{\varphi}), \\
& a^{-3} \Delta \mathcal{L}=\left[\sum_{n=1} \frac{1}{n !} \frac{\partial^{n} b_{3}^{I J K L}(\bar{\varphi})}{\partial\left(\bar{\varphi}_{M}\right)^{n}}\left(\delta \varphi_{M}\right)^{n}\right] \dot{\bar{\varphi}}_{I} \dot{\bar{\varphi}}_{J} \dot{\bar{\varphi}}_{K} \dot{\bar{\varphi}}_{L} \\
& -\left[\sum_{n=0} \frac{1}{n !} \frac{\partial^{n} b_{3}^{I J K L}(\bar{\varphi})}{\partial\left(\bar{\varphi}_{M}\right)^{n}}\left(\delta \varphi_{M}\right)^{n}\right] \dot{\bar{\varphi}}_{I} \dot{\bar{\varphi}}_{J}\left[-\dot{\bar{\varphi}}_{K} \dot{\delta \varphi_{L}}-\dot{\bar{\varphi}}_{L} \dot{\delta \varphi_{K}}-\dot{\delta} \dot{\varphi}_{K} \dot{\delta} \varphi_{L}+a^{-2} \partial_{i} \delta \varphi_{K} \partial^{i} \delta \varphi_{L}\right] \\
& -\left[\sum_{n=0} \frac{1}{n !} \frac{\partial^{n} b_{3}^{I J K L}(\bar{\varphi})}{\partial\left(\bar{\varphi}_{M}\right)^{n}}\left(\delta \varphi_{M}\right)^{n}\right]\left[-\dot{\bar{\varphi}}_{I} \dot{\delta \varphi_{J}}-\dot{\bar{\varphi}} \dot{\delta}_{\varphi_{I}}-\dot{\delta \varphi_{I}} \dot{\delta \varphi_{J}}+a^{-2} \partial_{i} \delta \varphi_{I} \partial^{i} \delta \varphi_{J}\right] \dot{\bar{\varphi}}_{K} \dot{\bar{\varphi}}_{L} \\
& +\left[\sum_{n=0} \frac{1}{n !} \frac{\partial^{n} b_{3}^{I J K L}(\bar{\varphi})}{\partial\left(\bar{\varphi}_{M}\right)^{n}}\left(\delta \varphi_{M}\right)^{n}\right] \times \\
& {\left[-\dot{\bar{\varphi}}_{I} \dot{\delta} \varphi_{J}-\dot{\bar{\varphi}}{ }_{J} \dot{\delta} \varphi_{I}-\dot{\delta} \varphi_{I} \dot{\delta} \varphi_{J}+a^{-2} \partial_{i} \delta \varphi_{I} \partial^{i} \delta \varphi_{J}\right]\left[-\dot{\bar{\varphi}}_{K} \dot{\delta} \varphi_{L}-\dot{\bar{\varphi}}_{L} \dot{\delta} \varphi_{K}-\dot{\delta} \varphi_{K} \dot{\delta} \varphi_{L}+a^{-2} \partial_{i} \delta \varphi_{K} \partial^{i} \delta \varphi_{L}\right]} \\
& -\frac{M^{2}}{2} \delta^{I J}\left[-2 \dot{\bar{\varphi}}_{I} \dot{\delta} \varphi_{J}-\dot{\delta} \dot{\varphi}_{I} \dot{\delta} \dot{\varphi}_{J}+a^{-2} \partial_{i} \delta \varphi_{I} \partial^{i} \delta \varphi_{J}\right]-M_{P}^{2}\left[\sum_{n=1} \frac{1}{n !} \frac{\partial^{n} U(\bar{\varphi})}{\partial\left(\bar{\varphi}_{M}\right)^{n}}\left(\delta \varphi_{M}\right)^{n}\right]
\end{aligned}
$$

where $a=a(t)$ is the scale factor of the FRW metric and $\partial_{i}$ are spatial derivatives. Note that the terms containing $\delta \varphi_{I}$ without any differentiations do not show themselves in the Lagrangian effectively. Since the $n^{\text {th }}$ equation of motion causes vanishing of the coefficients of $(n+1)^{t h}$ terms without any differentiation. Also the linear perturbation terms even including differentiation vanish because of the same reason. So effectively the Lagrangian for the perturbations is

$$
\begin{aligned}
a^{-3} \Delta \mathcal{L} & =b_{3}^{I J K L}(\bar{\varphi})\left\{\left[-\dot{\bar{\varphi}}_{I} \dot{\delta \varphi_{J}}-\dot{\bar{\varphi}}_{J} \dot{\delta} \varphi_{I}-\dot{\delta} \varphi_{I} \dot{\delta \varphi_{J}}+a^{-2} \partial_{i} \delta \varphi_{I} \partial^{i} \delta \varphi_{J}\right]\left[-\dot{\bar{\varphi}}_{K} \dot{\delta \varphi_{L}}-\dot{\bar{\varphi}}_{L} \dot{\delta \varphi_{K}}-\dot{\delta \varphi_{K}} \dot{\delta \varphi_{L}}+a^{-2} \partial_{i} \delta \varphi_{K} \partial^{i} \delta \varphi_{L}\right]\right. \\
& \left.-\dot{\bar{\varphi}}_{I} \dot{\bar{\varphi}}_{J}\left[-\dot{\delta} \dot{\varphi}_{K} \dot{\delta \varphi_{L}}+a^{-2} \partial_{i} \delta \varphi_{K} \partial^{i} \delta \varphi_{L}\right]-\left[-\dot{\delta \varphi_{I} \delta \varphi_{J}}+a^{-2} \partial_{i} \delta \varphi_{I} \partial^{i} \delta \varphi_{J}\right] \dot{\bar{\varphi}}_{K} \dot{\bar{\varphi}}_{L}\right\} \\
& -\frac{M^{2}}{2} \delta^{I J}\left[-\dot{\delta} \dot{\varphi}_{I} \dot{\delta} \varphi_{J}+a^{-2} \partial_{i} \delta \varphi_{I} \partial^{i} \delta \varphi_{J}\right]
\end{aligned}
$$

The second, third and fourth order of perturbations respectively can be represented as follows

$$
\begin{aligned}
& a^{-3} \Delta \mathcal{L}^{(2)}=b_{3}^{I J K L}(\overline{\bar{\varphi}})\left\{\dot{\bar{\varphi}}_{I} \dot{\bar{\varphi}}_{J} \dot{\delta} \varphi_{K} \dot{\delta \varphi_{L}}+\dot{\bar{\varphi}}_{I} \dot{\bar{\varphi}}_{K} \dot{\delta} \dot{\varphi}_{J} \dot{\delta \varphi_{L}}+\dot{\bar{\varphi}}_{I} \dot{\bar{\varphi}}_{L} \dot{\delta} \varphi_{K} \dot{\delta \varphi_{J}}+\dot{\bar{\varphi}}_{K} \dot{\bar{\varphi}}_{J} \dot{\delta} \dot{\varphi}_{I} \dot{\delta} \varphi_{L}+\dot{\bar{\varphi}}_{L} \dot{\bar{\varphi}}_{J} \dot{\delta} \dot{\varphi}_{I} \dot{\delta} \dot{\varphi}_{L}+\dot{\bar{\varphi}} K \dot{\bar{\varphi}}_{L} \dot{\delta} \varphi_{I} \dot{\delta} \varphi_{J}\right. \\
& \left.-a^{-2} \dot{\bar{\varphi}}_{I} \dot{\bar{\varphi}}_{J} \partial_{i} \delta \varphi_{K} \partial^{i} \delta \varphi_{L}-a^{-2} \dot{\bar{\varphi}}_{K} \dot{\bar{\varphi}}_{L} \partial_{i} \delta \varphi_{I} \partial^{i} \delta \varphi_{J}\right\}-\frac{M^{2}}{2} \delta^{I J}\left[-\dot{\delta} \varphi_{I} \dot{\delta} \varphi_{J}+a^{-2} \partial_{i} \delta \varphi_{I} \partial^{i} \delta \varphi_{J}\right]
\end{aligned}
$$$$
a^{-3} \Delta \mathcal{L}^{(3)}=b_{3}^{I J K L}(\bar{\varphi})\left\{\dot{\bar{\varphi}}_{I} \dot{\delta} \varphi_{J} \dot{\delta} \varphi_{K} \dot{\delta} \varphi_{L}+\dot{\bar{\varphi}}_{J} \dot{\delta} \dot{\varphi}_{I} \dot{\delta} \varphi_{K} \dot{\delta} \varphi_{L}+\dot{\bar{\varphi}}_{K} \dot{\delta} \varphi_{L} \dot{\delta} \dot{\varphi}_{I} \dot{\delta} \varphi_{J}+\dot{\bar{\varphi}}_{L} \dot{\delta} \dot{\varphi}_{K} \dot{\delta} \varphi_{I} \dot{\delta} \varphi_{J}\right.
$$$$
\left.-a^{-2}\left(\dot{\bar{\varphi}}_{I} \dot{\delta} \varphi_{J} \partial_{i} \delta \varphi_{K} \partial^{i} \delta \varphi_{L}+\dot{\bar{\varphi}}_{J} \dot{\delta} \varphi_{I} \partial_{i} \delta \varphi_{K} \partial^{i} \delta \varphi_{L}+\dot{\bar{\varphi}}_{K} \dot{\delta} \varphi_{L} \partial_{i} \delta \varphi_{I} \partial^{i} \delta \varphi_{J}+\dot{\bar{\varphi}}_{L} \dot{\delta} \varphi_{K} \partial_{i} \delta \varphi_{I} \partial^{i} \delta \varphi_{J}\right)\right\}
$$$$
a^{-3} \Delta \mathcal{L}^{(4)}=b_{3}^{I J K L}(\bar{\varphi})\left\{\dot{\delta} \varphi_{I} \dot{\delta} \varphi_{J} \dot{\delta} \varphi_{K} \dot{\delta} \varphi_{L}-a^{-2}\left(\dot{\delta} \varphi_{I} \dot{\delta} \varphi_{J} \partial_{i} \delta \varphi_{K} \partial^{i} \delta \varphi_{L}+\dot{\delta} \varphi_{K} \dot{\delta} \varphi_{L} \partial_{i} \delta \varphi_{I} \partial^{i} \delta \varphi_{J}\right)\right.
$$$$
\left.+a^{-4} \partial_{i} \delta \varphi_{I} \partial^{i} \delta \varphi_{J} \partial_{j} \delta \varphi_{K} \partial^{j} \delta \varphi_{L}\right\}
$$ 
Note that the above result exactly reduces to single field result in (3) with $b_{3}^{I J K L}(\bar{\varphi})=f(\bar{\varphi})$ 21]. It is obvious from (5) that the speed of sound is not one in the presence of non-vanishing $b_{3}^{I J K L}(\bar{\varphi})$. Note that due to $b_{3}^{I J K L}(\bar{\varphi})$ the cubic and quartic terms are appeared. This feature is in disagreement with [2, 4]. In their work the coefficient which displays $c_{s}$ just connects to the cubic term. But here it connects to the fourth order term too. In the next section we restrict the model to a two-field model. This makes it possible to study the adiabatic and entropy perturbations in more details without loss of generality in the main results.

\section{A SPECIFIC CASE: ADIABATIC VERSUS ENTROPY PERTURBATION}

In this section we re-write the above formalism in the language of adiabatic and entropy perturbations for a twofield model. This is crucial in this approach since in contrast to 44 here the adiabatic perturbation is not initially supposed. In [4] the additional perturbations are added to a model already containing the adiabatic perturbation i.e. [2]. In [4] the Goldstone boson, introduced in [2], plays the role of the adiabatic perturbation and the additional fields are employed as the entropy perturbations. But in our model there is no initially difference between $\varphi_{I}$ 's and consequently $\delta \varphi_{I}$ 's. So it is critical to distinguish between adiabatic and entropy modes to manifest their different physical meanings.

\section{A. The Most General Two-Field Model}

In this subsection we re-do perturbation calculations for a two-field model. To do this we start with (4) for two fields named $\varphi$ and $\chi$

$$
\begin{aligned}
\mathcal{L}=-a^{3}\{ & -\frac{M_{1}^{2}}{2} \partial_{\mu} \varphi \partial^{\mu} \varphi-\frac{M_{2}^{2}}{2} \partial_{\mu} \chi \partial^{\mu} \chi-M_{P}^{2} U(\varphi, \chi)+g_{1}(\varphi, \chi)\left(\partial_{\mu} \varphi \partial^{\mu} \varphi\right)^{2}+g_{2}(\varphi, \chi)\left(\partial_{\mu} \chi \partial^{\mu} \chi\right)^{2} \\
& +g_{3}(\varphi, \chi)\left(\partial_{\mu} \varphi \partial^{\mu} \varphi\right)\left(\partial_{\nu} \varphi \partial^{\nu} \chi\right)+g_{4}(\varphi, \chi)\left(\partial_{\mu} \chi \partial^{\mu} \chi\right)\left(\partial_{\nu} \chi \partial^{\nu} \varphi\right) \\
& \left.+g_{5}(\varphi, \chi)\left(\partial_{\mu} \varphi \partial^{\mu} \varphi\right)\left(\partial_{\nu} \chi \partial^{\nu} \chi\right)+g_{6}(\varphi, \chi)\left(\partial_{\mu} \varphi \partial^{\mu} \chi\right)\left(\partial_{\nu} \varphi \partial^{\nu} \chi\right)\right\}
\end{aligned}
$$

where $a=a(t)$ is the scale factor and $g_{i}$ 's are some arbitrary dimensionless and order one functions as mentioned before. By assuming $\varphi=\bar{\varphi}+\delta \varphi$ and $\chi=\bar{\chi}+\delta \chi$ the above Lagrangian reduces to

$$
\begin{aligned}
a^{-3} \mathcal{L}_{0} & =\left\{-\frac{M_{1}^{2}}{2} \partial_{\mu} \bar{\varphi} \partial^{\mu} \bar{\varphi}-\frac{M_{2}^{2}}{2} \partial_{\mu} \bar{\chi} \partial^{\mu} \bar{\chi}-M_{P}^{2} U(\bar{\varphi}, \bar{\chi})+g_{1}\left(\partial_{\mu} \bar{\varphi} \partial^{\mu} \bar{\varphi}\right)^{2}+g_{2}\left(\partial_{\mu} \bar{\chi} \partial^{\mu} \bar{\chi}\right)^{2}\right. \\
& \left.+g_{3}\left(\partial_{\mu} \bar{\varphi} \partial^{\mu} \bar{\varphi}\right)\left(\partial_{\nu} \bar{\varphi} \partial^{\nu} \bar{\chi}\right)+g_{4}\left(\partial_{\mu} \bar{\chi} \partial^{\mu} \bar{\chi}\right)\left(\partial_{\nu} \bar{\chi} \partial^{\nu} \bar{\varphi}\right)+g_{5}\left(\partial_{\mu} \bar{\varphi} \partial^{\mu} \bar{\varphi}\right)\left(\partial_{\nu} \bar{\chi} \partial^{\nu} \bar{\chi}\right)+g_{6}\left(\partial_{\mu} \bar{\varphi} \partial^{\mu} \bar{\chi}\right)\left(\partial_{\nu} \bar{\varphi} \partial^{\nu} \bar{\chi}\right)\right\} \\
& =\frac{M_{1}}{2} \dot{\bar{\varphi}}^{2}+\frac{M_{2}}{2} \dot{\bar{\chi}}^{2}-M_{P}^{2} U(\bar{\varphi}, \bar{\chi})+g_{1} \dot{\bar{\varphi}}^{4}+g_{2} \dot{\bar{\chi}}^{4}+g_{3} \dot{\bar{\varphi}}^{3} \dot{\bar{\chi}}+g_{4} \dot{\bar{\varphi}} \dot{\bar{\chi}}^{3}+\left(g_{5}+g_{6}\right) \dot{\bar{\varphi}}^{2} \dot{\bar{\chi}}^{2}
\end{aligned}
$$

for the background part. The corresponding equations of motion for $\bar{\varphi}$ reads as

$$
\begin{aligned}
& \frac{d}{d t}\left[M_{1} \dot{\bar{\varphi}}+4 g_{1} \dot{\bar{\varphi}}^{3}+3 g_{3} \dot{\bar{\varphi}}^{2} \dot{\bar{\chi}}+g_{4} \dot{\bar{\chi}}^{3}+2\left(g_{5}+g_{6}\right) \dot{\bar{\varphi}}_{\overline{\bar{\chi}}}^{2}\right]+3 H\left[M_{1} \dot{\bar{\varphi}}+4 g_{1} \dot{\bar{\varphi}}^{3}+3 g_{3} \dot{\bar{\varphi}}^{2} \dot{\bar{\chi}}+g_{4} \dot{\bar{\chi}}^{3}+2\left(g_{5}+g_{6}\right) \dot{\bar{\varphi}} \dot{\bar{\chi}}^{2}\right] \\
& +M_{P}^{2} U^{\prime}-\left(g_{1}^{\prime} \dot{\bar{\varphi}}^{4}+g_{2}^{\prime} \dot{\bar{\chi}}^{4}+g_{3}^{\prime} \dot{\bar{\varphi}}^{3} \dot{\bar{\chi}}+g_{4}^{\prime} \dot{\bar{\varphi}}^{3} \dot{\bar{\chi}}^{3}+\left(g_{5}^{\prime}+g_{6}^{\prime}\right) \dot{\bar{\varphi}}^{2} \dot{\bar{\chi}}^{2}\right)=0
\end{aligned}
$$

where $^{\prime}$ is the differentiation with respect to $\bar{\varphi}$ and the similar equation is true for $\bar{\chi}$. It is straightforward but messy to show that the above equation of motion (as well as $\bar{\chi}$ 's) for the background causes the Lagrangian of the first order perturbation becomes vanishing. So the non-trivial terms start from the second order perturbations succeeding with the third and the fourth order terms 22 for (8) as the following

$$
\begin{aligned}
a^{-3} \Delta \mathcal{L}^{(2)} & =\dot{\delta \varphi} \dot{\varphi}^{2}\left[\frac{M_{1}^{2}}{2}+6 g_{1} \dot{\bar{\varphi}}^{2}+3 g_{3} \dot{\bar{\varphi}} \dot{\bar{\chi}}+\left(g_{5}+g_{6}\right) \dot{\bar{\chi}}^{2}\right]+\dot{\delta} \dot{\chi}^{2}\left[\frac{M_{2}^{2}}{2}+6 g_{2} \dot{\bar{\chi}}^{2}+3 g_{4} \dot{\bar{\varphi}} \dot{\bar{\chi}}+\left(g_{5}+g_{6}\right) \dot{\bar{\varphi}}^{2}\right] \\
& +\dot{\delta \varphi} \dot{\delta} \chi\left[3 g_{3} \dot{\bar{\varphi}}^{2}+3 g_{4} \dot{\bar{\chi}}^{2}+4\left(g_{5}+g_{6}\right) \dot{\bar{\varphi}} \dot{\bar{\chi}}\right] \\
& -a^{-2}\left(\partial_{i} \delta \varphi \partial^{i} \delta \varphi\left[\frac{M_{1}}{2}+2 g_{1} \dot{\bar{\varphi}}^{2}+g_{3} \dot{\bar{\varphi}} \dot{\bar{\chi}}+g_{5} \dot{\bar{\chi}}^{2}\right]+\partial_{i} \delta \chi \partial^{i} \delta \chi\left[\frac{M_{2}}{2}+2 g_{2} \dot{\bar{\chi}}^{2}+g_{4} \dot{\bar{\varphi}} \dot{\bar{\chi}}+g_{5} \dot{\bar{\varphi}}^{2}\right]\right. \\
& \left.+\partial_{i} \delta \varphi \partial^{i} \delta \chi\left[g_{3} \dot{\bar{\varphi}}^{2}+g_{4} \dot{\bar{\chi}}^{2}+2 g_{6} \dot{\bar{\varphi}} \dot{\bar{\chi}}\right]\right)
\end{aligned}
$$




$$
\begin{aligned}
& a^{-3} \Delta \mathcal{L}^{(3)}=\dot{\delta} \dot{\varphi}^{3}\left[4 g_{1} \dot{\bar{\varphi}}+g_{3} \dot{\bar{\chi}}\right]+\dot{\delta \chi^{3}}\left[4 g_{2} \dot{\bar{\chi}}+g_{4} \dot{\bar{\varphi}}\right]+\dot{\delta \varphi} \dot{\varphi}^{2} \dot{\delta \chi}\left[3 g_{3} \dot{\bar{\varphi}}+2\left(g_{5}+g_{6}\right) \dot{\bar{\chi}}\right]+\dot{\delta} \dot{\varphi} \dot{\chi}{ }^{2}\left[3 g_{4} \dot{\bar{\chi}}+2\left(g_{5}+g_{6}\right) \dot{\bar{\varphi}}\right](11) \\
& -a^{-2}\left(\dot{\delta} \varphi \partial_{i} \delta \varphi \partial^{i} \delta \varphi\left[4 g_{1} \dot{\bar{\varphi}}+g_{3} \dot{\bar{\chi}}\right]+\dot{\delta} \chi \partial_{i} \delta \chi \partial^{i} \delta \chi\left[4 g_{2} \dot{\bar{\chi}}+g_{4} \dot{\bar{\varphi}}\right]+\dot{\delta} \varphi \partial_{i} \delta \chi \partial^{i} \delta \chi\left[g_{4} \dot{\bar{\chi}}+2 g_{5} \dot{\bar{\varphi}}\right]\right. \\
& \left.+\dot{\delta} \chi \partial_{i} \delta \varphi \partial^{i} \delta \varphi\left[g_{3} \dot{\bar{\varphi}}+2 g_{5} \dot{\bar{\chi}}\right]+\dot{\delta} \varphi \partial_{i} \delta \varphi \partial^{i} \delta \chi\left[2 g_{3} \dot{\bar{\varphi}}+2 g_{6} \dot{\bar{\chi}}\right]+\dot{\delta} \chi \partial_{i} \delta \varphi \partial^{i} \delta \chi\left[2 g_{4} \dot{\bar{\chi}}+2 g_{6} \dot{\bar{\varphi}}\right]\right)
\end{aligned}
$$$$
a^{-3} \Delta \mathcal{L}^{(4)}=g_{1} \dot{\delta \varphi}{ }^{4}+g_{2} \dot{\delta \chi}{ }^{4}+g_{3} \dot{\delta}{ }^{3} \dot{\delta} \dot{\chi}+g_{4} \dot{\delta} \dot{\chi}^{3} \dot{\delta} \varphi+\left(g_{5}+g_{6}\right) \dot{\delta \varphi} \dot{\varphi}^{2} \chi^{2}
$$$$
-a^{-2}\left(2 g_{1} \dot{\delta} \dot{\varphi}^{2} \partial_{i} \delta \varphi \partial^{i} \delta \varphi+2 g_{2} \dot{\delta} \chi^{2} \partial_{i} \delta \chi \partial^{i} \delta \chi+g_{3} \dot{\delta} \dot{\varphi}^{2} \partial_{i} \delta \varphi \partial^{i} \delta \chi+g_{4} \dot{\delta}{ }^{2} \partial_{i} \delta \chi \partial^{i} \delta \varphi\right.
$$$$
\left.+g_{3} \dot{\delta} \dot{\varphi} \dot{\bar{\chi}} \partial_{i} \delta \varphi \partial^{i} \delta \varphi+g_{4} \dot{\delta \chi} \dot{\bar{\delta} \varphi} \partial_{i} \delta \chi \partial^{i} \delta \chi+g_{5}\left(\dot{\delta \chi}{ }^{2} \partial_{i} \delta \varphi \partial^{i} \delta \varphi+\dot{\delta \varphi^{2}} \partial_{i} \delta \chi \partial^{i} \delta \chi\right)+2 g_{6} \dot{\delta} \dot{\varphi} \dot{\chi} \partial_{i} \delta \varphi \partial^{i} \delta \chi\right)
$$$$
+a^{-4}\left(g_{1}\left(\partial_{i} \delta \varphi \partial^{i} \delta \varphi\right)^{2}+g_{2}\left(\partial_{i} \delta \chi \partial^{i} \delta \chi\right)^{2}+g_{3} \partial_{i} \delta \varphi \partial^{i} \delta \varphi \partial_{j} \delta \chi \partial^{j} \delta \varphi+g_{4} \partial_{i} \delta \chi \partial^{i} \delta \chi \partial_{j} \delta \chi \partial^{j} \delta \varphi\right.
$$$$
\left.+g_{5} \partial_{i} \delta \varphi \partial^{i} \delta \varphi \partial_{j} \delta \chi \partial^{j} \delta \chi+g_{6}\left(\partial_{i} \delta \varphi \partial^{i} \delta \chi\right)^{2}\right) .
$$

It is interesting to mention that for $\varphi=\chi$, i.e. going back to one field case, all the above relations reduce to (3) with $f=g_{1}+g_{2}+g_{3}+g_{4}+g_{5}+g_{6}$ as it was expected.

\section{B. Adiabatic vs. Entropy Modes}

Now let us re-write the above terms in the language of adiabatic and entropy perturbations. The adiabatic perturbation is along the classical path and the entropy perturbation is orthogonal to it. Due to [10] they can be defined as follows

$$
\delta \sigma \equiv \vec{T} \cdot \vec{\delta}, \quad \delta s \equiv \vec{N} \cdot \vec{\delta}
$$

where $\delta \sigma$ and $\delta s$ are the adiabatic and entropy modes respectively and

$$
\vec{\delta} \equiv(\delta \varphi, \delta \chi), \quad \vec{T}=(\cos \theta, \sin \theta) \equiv(\dot{\varphi} / \dot{\sigma}, \dot{\chi} / \dot{\sigma}), \quad \vec{N} \equiv(\sin \theta,-\cos \theta)
$$

where $\dot{\sigma}^{2}=\dot{\varphi}^{2}+\dot{\chi}^{2}$. One can show easily

$$
\dot{\delta \varphi}=\cos \theta \vec{T} \cdot \dot{\vec{\delta}}+\sin \theta \vec{N} \cdot \dot{\vec{\delta}}, \quad \dot{\delta} \chi=\sin \theta \vec{T} \cdot \dot{\vec{\delta}}-\cos \theta \vec{N} \cdot \dot{\vec{\delta}}
$$

and it is easy to see that

$$
\dot{\delta \sigma}=\dot{\theta} \delta s+\vec{T} \cdot \dot{\vec{\delta}}, \quad \dot{\delta s}=-\dot{\theta} \delta \sigma+\vec{N} \cdot \dot{\vec{\delta}}
$$

and due to above relations

$$
\partial_{i} \delta \varphi=\cos \theta \quad \partial_{i} \delta \sigma+\sin \theta \quad \partial_{i} \delta s, \quad \partial_{i} \delta \chi=\sin \theta \quad \partial_{i} \delta \sigma-\cos \theta \quad \partial_{i} \delta s .
$$

Now by the above definitions we re-write the results of previous sub-section by plugging $\delta \sigma$ and $\delta s$ in. Let's start with the kinetic terms for leading order term in (10)

$$
\begin{aligned}
& \frac{M_{1}^{2}}{2} \dot{\delta} \dot{\varphi}^{2}+\frac{M_{2}^{2}}{2} \dot{\delta \dot{\chi}}{ }^{2}= \\
& \frac{M_{1}^{2}+M_{2}^{2}}{4}\left[\dot{\delta \varphi}^{2}+\dot{\delta} \dot{\chi}^{2}\right]+\frac{M_{1}^{2}-M_{2}^{2}}{4}\left[\dot{\delta}^{2}-\dot{\delta} \chi^{2}\right]= \\
& \frac{M_{1}^{2}+M_{2}^{2}}{4}\left[(\vec{T} \cdot \dot{\vec{\delta}})^{2}+(\vec{N} \cdot \dot{\vec{\delta}})^{2}\right]+\frac{M_{1}^{2}-M_{2}^{2}}{4}\left[\left(\cos ^{2} \theta-\sin ^{2} \theta\right)\left((\vec{T} \cdot \dot{\vec{\delta}})^{2}-(\vec{N} \cdot \dot{\vec{\delta}})^{2}\right)+4 \sin \theta \cos \theta T \cdot \dot{\vec{\delta}} N \cdot \dot{\vec{\delta}}\right]
\end{aligned}
$$


the same procedure is applicable for $a^{-2}\left(\frac{M_{1}^{2}}{2} \partial_{i} \delta \varphi \partial^{i} \delta \varphi+\frac{M_{2}^{2}}{2} \partial_{i} \delta \chi \partial^{i} \delta \chi\right)$ in (10). Now let us assume $M_{1}=M_{2}=M$ to make it comparable to results in [10]. In this case

$$
\begin{aligned}
\frac{1}{M^{2}} a^{-3} \mathcal{L}= & \frac{1}{2} \dot{\delta \varphi}{ }^{2}+\frac{1}{2} \dot{\delta \chi^{2}}-a^{-2}\left(\frac{1}{2} \partial_{i} \delta \varphi \partial^{i} \delta \varphi+\frac{1}{2} \partial_{i} \delta \chi \partial^{i} \delta \chi\right)= \\
& \frac{1}{2}\left[(\vec{T} \cdot \dot{\vec{\delta}})^{2}+(\vec{N} . \dot{\vec{\delta}})^{2}\right]-\frac{1}{2} a^{-2}\left(\partial_{i} \delta \sigma \partial^{i} \delta \sigma+\partial_{i} \delta s \partial^{i} \delta s\right)= \\
& \frac{1}{2}\left[(\dot{\delta \sigma}-\dot{\theta} \delta s)^{2}+(\dot{\delta} s+\dot{\theta} \delta \sigma)^{2}\right]-\frac{1}{2} a^{-2}\left(\partial_{i} \delta \sigma \partial^{i} \delta \sigma+\partial_{i} \delta s \partial^{i} \delta s\right)= \\
& \frac{1}{2}\left[\dot{\delta \sigma}^{2}+\dot{\theta}^{2} \delta s^{2}-2 \dot{\theta} \delta s \dot{\delta} \sigma+\dot{\delta s}^{2}+\dot{\theta}^{2} \delta \sigma^{2}+2 \dot{\theta} \delta \sigma \dot{\delta} s\right]-\frac{1}{2} a^{-2}\left(\partial_{i} \delta \sigma \partial^{i} \delta \sigma+\partial_{i} \delta s \partial^{i} \delta s\right)
\end{aligned}
$$

According to the above Lagrangian the equations of motion for $\delta \sigma$ and $\delta s$ become 23$]$

$$
\begin{aligned}
& \ddot{\delta \sigma}+3 H \dot{\delta \sigma}-a^{-2} \partial^{i} \partial_{i} \delta \sigma+\left(V_{\sigma \sigma}-\dot{\theta}^{2}\right) \delta \sigma=2 \dot{\theta} \dot{\delta s}+\left(\ddot{\theta}+3 H \dot{\theta}-V_{\sigma s}\right) \delta s \\
& \ddot{\delta s}+3 H \dot{\delta s}-a^{-2} \partial^{i} \partial_{i} \delta s+\left(V_{s s}-\dot{\theta}^{2}\right) \delta s=-2 \dot{\theta} \dot{\delta} \sigma-\left(\ddot{\theta}+3 H \dot{\theta}+V_{\sigma s}\right) \delta \sigma
\end{aligned}
$$

where $V_{\sigma s}=\left(\cos ^{2} \theta-\sin ^{2} \theta\right) V_{\varphi \chi}+\sin \theta \cos \theta\left(V_{\chi \chi}-V_{\varphi \varphi}\right)$. The above results are exactly same as (47) and (48) in [10] when ignoring metric perturbations, see Appendix B. Now let us do the same procedure for the second order perturbations due to the first order correction term in (10). At the first the terms containing time derivative

$$
\begin{aligned}
& \dot{\delta \varphi^{2}}\left[6 g_{1} \dot{\bar{\varphi}}^{2}+3 g_{3} \dot{\bar{\varphi}} \dot{\bar{\chi}}+\left(g_{5}+g_{6}\right) \dot{\bar{\chi}}^{2}\right]+\dot{\delta \chi^{2}}\left[6 g_{2} \dot{\bar{\chi}}^{2}+3 g_{4} \dot{\bar{\varphi}} \dot{\bar{\chi}}+\left(g_{5}+g_{6}\right) \dot{\bar{\varphi}}^{2}\right]+\dot{\delta \varphi} \dot{\delta \chi}\left[3 g_{3} \dot{\bar{\varphi}}^{2}+3 g_{4} \dot{\bar{\chi}}^{2}+4\left(g_{5}+g_{6}\right) \dot{\bar{\varphi}} \dot{\bar{\chi}}\right] \\
& =(\cos \theta \vec{T} \cdot \dot{\vec{\delta}}+\sin \theta \vec{N} \cdot \dot{\vec{\delta}})^{2}\left[6 g_{1} \dot{\bar{\varphi}}^{2}+3 g_{3} \dot{\bar{\varphi}} \dot{\bar{\chi}}+\left(g_{5}+g_{6}\right) \dot{\bar{\chi}}^{2}\right]+\left(\sin \theta \vec{T} \cdot \dot{\vec{\delta}}-\cos \theta \vec{N} \cdot \dot{\vec{j}}^{2}\left[6 g_{2} \dot{\bar{\chi}}^{2}+3 g_{4} \dot{\bar{\varphi}} \dot{\bar{\chi}}+\left(g_{5}+g_{6}\right) \dot{\bar{\varphi}} 2\right]\right. \\
& +\left(\begin{array}{llll}
\cos \theta & \vec{T} \cdot \dot{\vec{\delta}}+\sin \theta & \vec{N} \cdot \dot{\vec{\delta}}
\end{array}\right)\left(\begin{array}{ccc}
\sin \theta & \vec{T} \cdot \dot{\vec{\delta}}-\cos \theta & \vec{N} \cdot \dot{\vec{\delta}}
\end{array}\right)\left[3 g_{3} \dot{\bar{\varphi}}^{2}+3 g_{4} \dot{\bar{\chi}}^{2}+4\left(g_{5}+g_{6}\right) \dot{\dot{\varphi}} \dot{\bar{\chi}}\right] \\
& =(\vec{T} \cdot \dot{\vec{\delta}})^{2} \times\left\{\cos ^{2} \theta\left[6 g_{1} \dot{\bar{\varphi}}^{2}+3 g_{3} \dot{\bar{\varphi}} \dot{\bar{\chi}}+\left(g_{5}+g_{6}\right) \dot{\bar{\chi}}^{2}\right]+\sin ^{2} \theta\left[6 g_{2} \dot{\bar{\chi}}^{2}+3 g_{4} \dot{\bar{\varphi}} \dot{\bar{\chi}}+\left(g_{5}+g_{6}\right) \dot{\bar{\varphi}}^{2}\right]\right. \\
& \left.+\sin \theta \cos \theta\left[3 g_{3} \dot{\bar{\varphi}}^{2}+3 g_{4} \dot{\bar{\chi}}^{2}+4\left(g_{5}+g_{6}\right) \dot{\bar{\varphi}} \dot{\bar{\chi}}\right]\right\} \\
& +(\vec{N} \cdot \dot{\vec{\delta}})^{2} \times\left\{\sin ^{2} \theta\left[6 g_{1} \dot{\bar{\varphi}}^{2}+3 g_{3} \dot{\bar{\varphi}} \dot{\bar{\chi}}+\left(g_{5}+g_{6}\right) \dot{\bar{\chi}}^{2}\right]+\cos ^{2} \theta\left[6 g_{2} \dot{\bar{\chi}}^{2}+3 g_{4} \dot{\bar{\varphi}} \dot{\bar{\chi}}+\left(g_{5}+g_{6}\right) \dot{\bar{\varphi}}^{2}\right]\right. \\
& \left.-\sin \theta \cos \theta\left[3 g_{3} \dot{\bar{\varphi}}^{2}+3 g_{4} \dot{\bar{\chi}}^{2}+4\left(g_{5}+g_{6}\right) \dot{\bar{\varphi}} \dot{\bar{\chi}}\right]\right\} \\
& +(\vec{T} . \dot{\vec{\delta}})(\vec{N} . \dot{\vec{\delta}}) \times\left\{2 \cos \theta \sin \theta\left(\left[6 g_{1} \dot{\bar{\varphi}}^{2}+3 g_{3} \dot{\bar{\varphi}} \dot{\bar{\chi}}+\left(g_{5}+g_{6}\right) \dot{\bar{\chi}}^{2}\right]-\left[6 g_{2} \dot{\bar{\chi}}^{2}+3 g_{4} \dot{\bar{\varphi}} \dot{\bar{\chi}}+\left(g_{5}+g_{6}\right) \dot{\bar{\varphi}}^{2}\right]\right)\right. \\
& \left.+\left(\sin ^{2} \theta-\cos ^{2} \theta\right)\left[3 g_{3} \dot{\bar{\varphi}}^{2}+3 g_{4} \dot{\bar{\chi}}^{2}+4\left(g_{5}+g_{6}\right) \dot{\bar{\varphi}} \dot{\bar{\chi}}\right]\right\}
\end{aligned}
$$

which can be re-written as the following 24 ]

$$
\begin{aligned}
& 6 \dot{\sigma}^{2}(\vec{T} \cdot \dot{\vec{\delta}})^{2} \times\left[g_{1} \cos ^{4} \theta+g_{2} \sin ^{4} \theta+g_{3} \cos ^{3} \theta \sin \theta+g_{4} \cos \theta \sin ^{3} \theta+\left(g_{5}+g_{6}\right) \cos ^{2} \theta \sin ^{2} \theta\right] \\
+ & \dot{\sigma}^{2}(\vec{N} \cdot \dot{\vec{\delta}})^{2} \times \\
& {\left[\left(g_{5}+g_{6}\right)\left(\cos ^{4} \theta+\sin ^{4} \theta\right)+3\left(g_{4}-g_{3}\right)\left(\cos ^{3} \theta \sin \theta-\cos \theta \sin ^{3} \theta\right)+2\left(3\left(g_{1}+g_{2}\right)+2\left(g_{5}+g_{6}\right)\right) \cos ^{2} \theta \sin ^{2} \theta\right] } \\
+ & 3 \dot{\sigma}^{2}(\vec{T} \cdot \dot{\vec{\delta}})(\vec{N} \cdot \dot{\vec{\delta}}) \times \\
& {\left[-g_{3} \cos ^{4} \theta+g_{4} \sin ^{4} \theta+2\left(2 g_{1}-\left(g_{5}+g_{6}\right)\right) \cos ^{3} \theta \sin \theta-2\left(2 g_{2}+\left(g_{5}+g_{6}\right)\right) \cos \theta \sin ^{3} \theta+3\left(g_{3}-g_{4}\right) \cos ^{2} \theta \sin ^{2} \theta\right] }
\end{aligned}
$$


and similarly for the spatial differentiation

$$
\begin{aligned}
& -2 a^{-2} \dot{\sigma}^{2}\left(\partial_{i} \delta \sigma\right)^{2} \times\left[g_{1} \cos ^{4} \theta+g_{2} \sin ^{4} \theta+g_{3} \cos ^{3} \theta \sin \theta+g_{4} \cos \theta \sin ^{3} \theta+2\left(g_{5}+g_{6}\right) \cos ^{2} \theta \sin ^{2} \theta\right] \\
& -a^{-2} \dot{\sigma}^{2}\left(\partial_{i} \delta s\right)^{2} \times\left[g_{5}\left(\cos ^{4} \theta+\sin ^{4} \theta\right)+\left(g_{4}-g_{3}\right)\left(\cos ^{3} \theta \sin \theta-\cos \theta \sin ^{3} \theta\right)+2\left(g_{1}+g_{2}-g_{6}\right) \cos ^{2} \theta \sin ^{2} \theta\right] \\
& -a^{-2} \dot{\sigma}^{2} \partial_{i} \delta \sigma \partial^{i} \delta s \times \\
& {\left[-g_{3} \cos ^{4} \theta+g_{4} \sin ^{4} \theta+2\left(2 g_{1}-\left(g_{5}+g_{6}\right)\right) \cos ^{3} \theta \sin \theta-2\left(2 g_{2}+\left(g_{5}+g_{6}\right)\right) \cos \theta \sin ^{3} \theta+3\left(g_{3}-g_{4}\right) \cos ^{2} \theta \sin ^{2} \theta\right] .}
\end{aligned}
$$

Up to now we fully considered the second order perturbation terms in the language of adiabatic and entropy modes. In quadratic level the speed of sound is a matter of interest hence it is noteworthy to take a look at it. It is obvious to the above relations that $\delta \sigma$ and $\delta s$ can have different speeds of sound generally. To have a sense about it let's consider a special case that $\delta \sigma$ has $c_{s}=1$ and $\delta s$ has $c_{s} \neq 1$. To see this, assume the special case with $g_{1}=g_{2}=g_{3}=g_{4}=0$ and $g_{5}+g_{6}=0$. In this case the coefficients of $\dot{\delta \sigma^{2}}$ and $\left(\partial_{i} \delta \sigma\right)^{2}$ are same and result in $c_{s}=1$ for $\delta \sigma[25$. But the coefficient of $\dot{\delta s}^{2}$ is $\frac{M^{2}}{2}$ and for $\left(\partial_{i} \delta s\right)^{2}$ is $-\frac{1}{2} a^{-2}\left(M^{2}-2 g_{5} \dot{\sigma}^{2}\right)$ that means $c_{s}^{2}=1-2 \frac{g_{5} \dot{\sigma}^{2}}{M^{2}}$. Note that here we write the $M$ explicitly to make the comparison of the terms easier. A characteristic property of $c_{s}$ is its $\dot{\sigma}^{2}$-dependence which seems interesting. However the effective field theory is valid where the correction terms are smaller than the leading terms in (44) to have an acceptable expansion i.e. $\frac{\left|b_{3}^{I J K L}(\bar{\varphi})\right| \dot{\sigma}^{2}}{M^{2}}<1$. Even more, $\frac{\left|b_{3}^{I J K L}(\bar{\varphi})\right| \dot{\sigma}^{2}}{M^{2}}<<1$ should be satisfied to make skipping higher order correction terms in (4) acceptable. So the speed of sound in this model is almost one. This fact shows that for the single field model the large non-Gaussinity is not expected. But in the following we will discuss on the case of multi-field models. In multi-field models even with $c_{s} \simeq 1$ the large non-Gaussianity can be occurred in some specific circumstances.

One can do this procedure for the higher order perturbation terms (11) and (12) which are the fundaments of studying non-Gaussianity for multi-field models. The results are as follows [26] for $a^{-3} \Delta \mathcal{L}^{(3)}$

$$
\begin{aligned}
& 4 \dot{\sigma}(\vec{T} \cdot \dot{\vec{\delta}})^{3} \times\left[g_{1} \cos ^{4} \theta+g_{2} \sin ^{4} \theta+g_{3} \cos ^{3} \theta \sin \theta+g_{4} \cos \theta \sin ^{3} \theta+\left(g_{5}+g_{6}\right) \cos ^{2} \theta \sin ^{2} \theta\right] \\
+ & \dot{\sigma}(\vec{N} \cdot \dot{\vec{\delta}})^{3} \times \\
& {\left[-g_{4} \cos ^{4} \theta+g_{3} \sin ^{4} \theta-2\left(2 g_{2}-\left(g_{5}+g_{6}\right)\right) \cos ^{3} \theta \sin \theta+2\left(2 g_{1}-\left(g_{5}+g_{6}\right)\right) \cos \theta \sin ^{3} \theta+3\left(g_{4}-g_{3}\right) \cos ^{2} \theta \sin ^{2} \theta\right] } \\
+ & 3 \dot{\sigma}(\vec{T} \cdot \dot{\vec{\delta}})^{2}(\vec{N} \cdot \dot{\vec{\delta}}) \times \\
& {\left[-g_{3} \cos ^{4} \theta+g_{4} \sin ^{4} \theta+2\left(2 g_{1}-\left(g_{5}+g_{6}\right)\right) \cos ^{3} \theta \sin \theta-2\left(2 g_{2}-\left(g_{5}+g_{6}\right)\right) \cos \theta \sin ^{3} \theta+3\left(g_{3}-g_{4}\right) \cos ^{2} \theta \sin ^{2} \theta\right] } \\
+ & 2 \dot{\sigma}(\vec{T} \cdot \dot{\vec{\delta}})(\vec{N} \cdot \dot{\vec{\delta}})^{2} \times \\
& {\left[\left(g_{5}+g_{6}\right)\left(\cos ^{4} \theta+\sin ^{4} \theta\right)+3\left(g_{4}-g_{3}\right)\left(\cos ^{3} \theta \sin \theta-\cos \theta \sin ^{3} \theta\right)+\left(3\left(g_{1}+g_{2}\right)-2\left(g_{5}+g_{6}\right)\right) \cos ^{2} \theta \sin ^{2} \theta\right] }
\end{aligned}
$$


and for $a^{-3} \Delta \mathcal{L}^{(4)}$ it becomes

$$
\begin{aligned}
& (\vec{T} \cdot \dot{\vec{\delta}})^{4} \times\left[g_{1} \cos ^{4} \theta+g_{2} \sin ^{4} \theta+g_{3} \cos ^{3} \theta \sin \theta+g_{4} \cos \theta \sin ^{3} \theta+\left(g_{5}+g_{6}\right) \cos ^{2} \theta \sin ^{2} \theta\right] \\
+ & (\vec{N} \cdot \dot{\vec{\delta}})^{4} \times\left[g_{2} \cos ^{4} \theta+g_{1} \sin ^{4} \theta-g_{4} \cos ^{3} \theta \sin \theta-g_{3} \cos \theta \sin ^{3} \theta+\left(g_{5}+g_{6}\right) \cos ^{2} \theta \sin ^{2} \theta\right] \\
+ & (\vec{T} \cdot \dot{\vec{\delta}})^{3}(\vec{N} \cdot \dot{\vec{\delta}}) \times \\
& {\left[-g_{3} \cos ^{4} \theta+g_{4} \sin ^{4} \theta+2\left(2 g_{1}-\left(g_{5}+g_{6}\right)\right) \cos ^{3} \theta \sin \theta-2\left(2 g_{2}-\left(g_{5}+g_{6}\right)\right) \cos \theta \sin ^{3} \theta+3\left(g_{3}-g_{4}\right) \cos ^{2} \theta \sin ^{2} \theta\right] } \\
+ & (\vec{T} \cdot \dot{\vec{\delta}})(\vec{N} \cdot \dot{\vec{\delta}})^{3} \times \\
& {\left[-g_{4} \cos ^{4} \theta+g_{3} \sin ^{4} \theta-2\left(2 g_{2}-\left(g_{5}+g_{6}\right)\right) \cos ^{3} \theta \sin \theta+2\left(2 g_{1}-\left(g_{5}+g_{6}\right)\right) \cos \theta \sin ^{3} \theta+3\left(g_{4}-g_{3}\right) \cos ^{2} \theta \sin ^{2} \theta\right] } \\
+ & (\vec{T} \cdot \dot{\vec{\delta}})^{2}(\vec{N} \cdot \dot{\vec{\delta}})^{2} \times \\
& {\left[\left(g_{5}+g_{6}\right)\left(\cos ^{4} \theta+\sin ^{4} \theta\right)+3\left(g_{4}-g_{3}\right)\left(\cos ^{3} \theta \sin \theta-\cos \theta \sin ^{3} \theta\right)+2\left(3\left(g_{1}+g_{2}\right)-2\left(g_{5}+g_{6}\right)\right) \cos ^{2} \theta \sin ^{2} \theta\right] . }
\end{aligned}
$$

Now let us just consider the terms containing $(\vec{T} . \dot{\vec{\delta}})$ and write them together as 27]

$$
4 \dot{\sigma}^{2}\left[g_{1} \cos ^{4} \theta+g_{2} \sin ^{4} \theta+g_{3} \cos ^{3} \theta \sin \theta+g_{4} \cos \theta \sin ^{3} \theta+\left(g_{5}+g_{6}\right) \cos ^{2} \theta \sin ^{2} \theta\right] \times\left((\vec{T} \cdot \dot{\vec{\delta}})^{2}+(\vec{T} \cdot \dot{\vec{\delta}})^{3} / \dot{\sigma}+\frac{1}{4}(\vec{T} \cdot \dot{\vec{\delta}})^{4} / \dot{\sigma}^{2}\right)
$$

Comparison the above relation with the relation in (3) manifests that $\sigma,[\ldots]$ and $(\vec{T} . \dot{\vec{\delta}})$ play the role of $\bar{\varphi}, f(\bar{\varphi})$ and $\dot{\delta \varphi}$ respectively. The significant property of this model is the appearance of $(\vec{T} . \dot{\vec{\delta}})$ and $(\vec{N} . \dot{\vec{\delta}})$ or equivalently $(\dot{\delta} \sigma-\dot{\theta} \delta s)$ and $(\dot{\delta s}+\dot{\theta} \delta \sigma)$ respectively. This means that $\dot{\delta \sigma}$ and $\delta s$ are always together and the same for $\dot{\delta s}$ and $\delta \sigma$. This characteristic feature of this model has some observational consequences which are discussed in the following.

\section{The Amplitude of Non-Gaussianity}

Now we are going to estimate the non-Gaussianity amplitude. To do this one procedure is comparison between the non-linear terms and the linear ones. Mathematically, the amplitude of non-Gaussianity $f_{N L}$, bi-spectrum, can be estimated as $\frac{\mathcal{L}^{(3)}}{\mathcal{L}^{(2)}} \times \zeta^{-1}$ where $\zeta$ is the curvature perturbation [6]. Note that the dominant amplitude of the terms containing time derivatives comes from their amplitude at horizon crossing. At this time $\frac{d}{d t} \sim H$ where $H$ is the Hubble constant. Hence for the second order perturbations the Lagrangian $\mathcal{L}^{(2)}$ can be written in an abstract form as $\left\{H^{2}, H \dot{\theta}, \dot{\theta}^{2}\right\} \times M^{2} \times \delta \sigma^{2}$. The same analyze for $\mathcal{L}^{(3)}$ results in $\left\{H^{3}, H^{2} \dot{\theta}, H \dot{\theta}^{2}, \dot{\theta}^{3}\right\} \times f\left(g_{i}\right) \times \dot{\sigma} \times \delta \sigma^{3}$. So in an abstract form

$$
\frac{\mathcal{L}^{(3)}}{\mathcal{L}^{(2)}}=\frac{\left\{H^{3}, H^{2} \dot{\theta}, H \dot{\theta}^{2}, \dot{\theta}^{3}\right\}}{\left\{H^{2}, H \dot{\theta}, \dot{\theta}^{2}\right\}} \times\left(\frac{f\left(g_{i}\right)}{M^{2}} \dot{\sigma}^{2}\right) \times \frac{\delta \sigma}{\dot{\sigma}}=\frac{\left\{H^{3}, H^{2} \dot{\theta}, H \dot{\theta}^{2}, \dot{\theta}^{3}\right\}}{H \times\left\{H^{2}, H \dot{\theta}, \dot{\theta}^{2}\right\}} \times\left(\frac{f\left(g_{i}\right)}{M^{2}} \dot{\sigma}^{2}\right) \times \zeta
$$

where $\zeta \sim \frac{H \delta \sigma}{\dot{\sigma}}$ is interpreted as curvature perturbation. Now we consider two different regimes $\dot{\theta}<<H$ and $\dot{\theta}>>H$. The first regime, $\dot{\theta}<<H$, physically means that the model is a single field model effectively. In this case the amplitude of bi-spectrum can be approximated by

$$
f_{N L} \sim \frac{\mathcal{L}^{(3)}}{\mathcal{L}^{(2)}} \zeta^{-1} \sim \frac{f\left(g_{i}\right)}{M^{2}} \dot{\sigma}^{2}
$$

but remember that the validity of the effective field theory imposes $\frac{f\left(g_{i}\right)}{M^{2}} \dot{\sigma}^{2}<1$. So in this case as mentioned before there is no significant non-Gaussianity which is in agreement with the single field models of inflation [11]. But the other case, $\dot{\theta}>>H$, means that the classical path in the phase space is highly curved [12]. In other words it means the classical path in the phase-space is far from a straight line $(\dot{\theta}=0)$. So the existence of the entropic field is unavoidable. For this case the amplitude of $f_{N L}$ is

$$
f_{N L} \sim \frac{\mathcal{L}^{(3)}}{\mathcal{L}^{(2)}} \zeta^{-1} \sim \frac{\dot{\theta}}{H} \times\left(\frac{f\left(g_{i}\right)}{M^{2}} \dot{\sigma}^{2}\right) .
$$


Now the factor $\frac{\dot{\theta}}{H} \times\left(\frac{f\left(g_{i}\right)}{M^{2}} \dot{\sigma}^{2}\right)$ can be large and results in large non-Gaussianity consequently. So the large curvature of the classical path in the phase-space results in the large non-Gaussianity. Though this result can be compared to the other works in the literature [9] and in the effective field theory context [5] but the curvature of the classical path is restricted due to observed scale invariant power spectrum [13].

For a moment let us relax the constraint on the $\frac{f\left(g_{i}\right)}{M^{2}} \dot{\sigma}^{2}$. Consequently the correction terms in (8) causes the large non-Gaussinity. However the relaxation of the constraint can be justified by assuming that our model is completely described by (8) without any higher order correction terms. This needs fine tuning which is not impossible but it is not natural. However there is another method to rationalize this assumption. Instead of fine tuning the model automatically shows this property via for example Vainshtein mechanism [14, 15].

The same is applicable for tri-spectrum by an estimation as $\tau_{N L} \sim \frac{\mathcal{L}^{(4)}}{\mathcal{L}^{(2)}} \times \zeta^{-2}$. The fourth order Lagrangian, $\mathcal{L}^{(4)}$, can be written in the abstract form as $\left\{H^{4}, H^{3} \dot{\theta}, H^{2} \dot{\theta}^{2}, H \dot{\theta}^{3}, H \dot{\theta}^{3}, \dot{\theta}^{4}\right\} \times f\left(g_{i}\right) \times \delta \sigma^{4}$ and then

$$
\tau_{N L} \sim \frac{\mathcal{L}^{(4)}}{\mathcal{L}^{(2)}} \zeta^{-2} \sim \frac{f\left(g_{i}\right)}{M^{2}} \dot{\sigma}^{2}
$$

for $\dot{\theta}<<H$ and

$$
\tau_{N L} \sim \frac{\mathcal{L}^{(4)}}{\mathcal{L}^{(2)}} \zeta^{-2} \sim\left(\frac{\dot{\theta}}{H}\right)^{2} \times\left(\frac{f\left(g_{i}\right)}{M^{2}} \dot{\sigma}^{2}\right)
$$

for $\dot{\theta}>>H$.

\section{The Shape of Non-Gaussianity}

Now let us focus on the shape of possible non-Gaussinity predicted by our model. In principle all the possible interaction terms between $\delta \sigma, \delta s$ and their derivatives exist in our model. This fact means all the non-Gaussianity shape can be produced. However the amplitude of different shapes are different. As a general argument it can be emphasized that for different regimes of $\frac{\dot{\theta}}{H}$ different shapes are dominant. For the case $\dot{\theta}<<H$ the terms containing time derivatives become dominated. This means in this limit the equilateral shape is the main one among the others. But it does not mean the other shapes do not exist i.e. the "Cosine" between different shapes are not zero. In the other limit, $\dot{\theta}>>H$, the terms without derivative become dominant and then the local shape is dominant. This result is in agreement with the prediction for multi-field inflation models [11]. Note that in [4] since the additional entropy perturbations are added by symmetry then they do not have any term without derivative in their Lagrangian. In this sense they do not predict a dominant local shape for their model which is in disagreement with our result.

A characteristic feature of this model is appearance of just two combinations of the fields i.e. $\vec{T} \cdot \dot{\vec{\delta}}$ and $\vec{N} \cdot \dot{\vec{\delta}}$ in all the terms including the second, third and fourth orders. To explain what is the physical result of this fact let us concentrate on $\vec{T} \cdot \dot{\vec{\delta}}=\dot{\delta} \sigma-\dot{\theta} \delta s$, as an example. The third order term of this combination is $(\vec{T} \cdot \dot{\vec{\delta}})^{3}=\delta \dot{\sigma}^{3}-3 \dot{\theta} \delta \dot{\sigma}^{2} \delta s+$ $3 \dot{\theta}^{2} \dot{\delta} \sigma \delta s^{2}-\dot{\theta}^{3} \delta s^{3}$. Without worrying about the amplitude in this part let us focus on the first and the last term. The definite prediction of this model is that if any equilateral non-Gaussianity due to the first term, i.e. $\delta \dot{\sigma}^{3}$, be observed then it has to be observed a local non-Gaussianity due to the last term[28], i.e. $\delta s^{3}$. So the non-Gaussianity predicted by this model cannot be pure e.g. pure equilateral shape. Hence mathematically, the "Cosine" between two shapes cannot be zero and more the "Cosine" depends on the $\dot{\theta}$ and is fixed by the model. This argument is true for the other third order terms as well as fourth order ones. To conclude, it seems that this model predicts a definite combination of different shapes for the non-Gaussianity if the amplitude allows to observe them.

\section{An Example}

In this subsection we are going to show how the general statements mentioned before do work in a simple example. Here we assume that all the $g_{i}$ 's vanish except $g_{1}(\varphi, \chi)[29]$ which is a generalization of the model in [7]. In addition we assume there is no potential term [30]. According to the background Lagrangian (9) the equations of motion for our special case become

$$
\begin{aligned}
& \ddot{\varphi}\left(1+12 \frac{g_{1}}{M^{2}} \dot{\varphi}^{2}\right)+3 H \dot{\varphi}\left(1+4 \frac{g_{1}}{M^{2}} \dot{\varphi}^{2}\right)+\frac{1}{M^{2}} \dot{\varphi}^{3}\left(3 \dot{\varphi} \frac{\partial g_{1}}{\partial \varphi}+4 \dot{\chi} \frac{\partial g_{1}}{\partial \chi}\right)=0 \\
& \ddot{\chi}+3 H \dot{\chi}-\frac{1}{M^{2}} \dot{\varphi}^{4} \frac{\partial g_{1}}{\partial \chi}=0
\end{aligned}
$$


where $M=M_{1}=M_{2}$ is assumed. On the other hand, what can cause the significant non-Gaussianity is $\dot{\theta}$ as mentioned before. In general due to the definition of $\theta$ in (14), $\dot{\theta}$ can be read as

$$
\dot{\theta}=\frac{-\ddot{\varphi} \dot{\chi}+\dot{\varphi} \ddot{\chi}}{\dot{\varphi}^{2}+\dot{\chi}^{2}}
$$

and in our special case by considering (25) it becomes (up to the first order of $g_{1} \frac{\dot{\varphi}^{2}}{M^{2}}$ )

$$
\frac{\dot{\theta}}{H} \simeq 24\left(-g_{1} \frac{\dot{\varphi}^{2}}{M^{2}}\right) \frac{\dot{\varphi} \dot{\chi}}{\dot{\varphi}^{2}+\dot{\chi}^{2}}+\frac{1}{H} \frac{1}{M^{2}} \frac{\dot{\varphi}^{3}}{\dot{\varphi}^{2}+\dot{\chi}^{2}}\left[3 \frac{\partial g_{1}}{\partial \varphi} \dot{\varphi} \dot{\chi}+\frac{\partial g_{1}}{\partial \chi}\left(\dot{\varphi}^{2}+4 \dot{\chi}^{2}\right)\right] \text {. }
$$

The condition $g_{1} \frac{\dot{\varphi}^{2}}{M_{1}^{2}}<1$ ensures the validity of the effective field theory. So it is not bad to estimate $24 g_{1} \frac{\dot{\varphi}^{2}}{M_{1}^{2}} \sim 1$. Then due to the first term $\frac{\dot{\theta}}{H} \sim \frac{\dot{\varphi} \dot{\chi}}{\dot{\varphi}^{2}+\dot{\chi}^{2}}$ which means the maximum of $f_{N L}$ in (22) is less than one. A successful inflation in the slow-roll regime restricts the value of the field velocities which may restrict more the above estimation. To discuss on the second term let us divide $g_{1}(\varphi, \chi)$ to its amplitude and functionality as $g_{1}(\varphi, \chi)=\left|g_{1}\right| \times f(\varphi, \chi)$ such that $|g|$ is the amplitude of the $g_{1}(\varphi, \chi)$ and $f(\varphi, \chi)$ represents its functional form. So the second term can be estimated as (by assuming $\dot{\varphi} \sim \dot{\chi}$ )

$$
\frac{\dot{\theta}}{H} \simeq\left(\left|g_{1}\right| \frac{\dot{\varphi}^{2}}{M^{2}}\right) \frac{\dot{\varphi}}{2 H}\left[3 \frac{\partial f}{\partial \varphi}+5 \frac{\partial f}{\partial \chi}\right]
$$

where $\left|g_{1}\right| \frac{\dot{\varphi}^{2}}{M^{2}}<1$ has to be satisfied. On the other hand one of the Friedmann equations (in the absence of the potential) is $H^{2}=\frac{M^{2}}{2} \dot{\varphi}^{2}+\frac{M^{2}}{2} \dot{\chi}^{2} \sim M^{2} \dot{\varphi}^{2}$ for the zeroth order of $g_{1} \frac{\dot{\varphi}^{2}}{M^{2}}$. Now if $\frac{\partial f}{\partial \varphi}$ or $\frac{\partial f}{\partial \chi}$ have the significant amplitude with respect to $M$ then a large amplitude of non-Gaussianity would be expected. This can be realized by assuming sharp features in the functionality of $g_{1}(\varphi, \chi)$ maybe due to a phase transition.

\section{Some Clarifications on Differences with Senatore and Zaldarriaga [4]}

The significant difference is the existence of the terms containing the adiabatic and entropy perturbations themselves not just their derivatives. The reason for this difference is in how the effective field theory is constructed in [4]. As mentioned before in their model the adiabatic mode is borrowed from [2] which satisfies a shift symmetry. Then the entropy modes are added and satisfy the shift symmetry too. Consequently, in their formalism they have just derivative of perturbations. But in contrast we do not start with distinguishable fields then we do not have any difference between the perturbations initially. So by this starting point we had to define the adiabatic and entropy perturbations. This is what has been done in this section in details. Now the question is that is there any special transformation for adiabatic and entropy perturbation in our model? Yes, it is locally rotated shift transformation i.e.

$$
\begin{array}{r}
\delta \sigma \rightarrow \delta \sigma+\left(c_{1} \cos \theta+c_{2} \sin \theta\right) \\
\delta s \rightarrow \delta s+\left(c_{1} \sin \theta-c_{2} \cos \theta\right)
\end{array}
$$

where $\theta=\arctan (\dot{\chi} / \dot{\varphi})$. Note that the rotational angle depends on the background fields time evolution. To achieve this result, the starting point is the Lagrangian for two fields i.e. the relations (10), (11) and (12). By looking at these relations it is obvious that $\delta \varphi \rightarrow \delta \varphi+c_{1}$ and $\delta \chi \rightarrow \delta \chi+c_{2}$ is a symmetry of the model where $c_{1}$ and $c_{2}$ are two independent arbitrary constants. So due to (13) one can get the above relation (26) as the corresponding transformation of $\delta \sigma$ and $\delta s$. According to this relation the invariant terms corresponding to $\dot{\delta \varphi}$ and $\dot{\delta \chi}$ are not $\dot{\delta \sigma}$ and $\dot{\delta s}$ but

$$
\begin{aligned}
& \dot{\delta \sigma}-\dot{\theta} \delta s \rightarrow \dot{\delta \sigma}-\dot{\theta} \delta s \\
& \dot{\delta s}+\dot{\theta} \delta \sigma \rightarrow \dot{\delta s}+\dot{\theta} \delta \sigma
\end{aligned}
$$

which are $\vec{T} . \dot{\vec{\delta}}$ and $\vec{N} . \dot{\vec{\delta}}$ respectively. Not surprisingly, these terms construct whole Lagrangian in adiabatic and entropy perturbations language as seen previously.

So it seems initially supposed adiabatic perturbation in 4] results in lack of all possible terms in the effective Lagrangian. Our proposition to solve this problem is based on the discussion in this subsection. The main building 
blocks for an effective field theory of multi-field inflation are not $\dot{\delta \sigma}$ and $\dot{\delta s}$ but they are $\vec{T} \cdot \dot{\vec{\delta}}$ and $\vec{N}$. $\dot{\vec{\delta}}$. So the most general Lagrangian for the perturbations in the multi-field context should be written as [31]

$$
\Delta \mathcal{L} \propto \sum_{m, n} c_{m n}(\vec{T} . \dot{\vec{\delta}})^{m}(\vec{N} \cdot \dot{\vec{\delta}})^{n}
$$

for arbitrary $c_{m n}$. The above Lagrangian can be considered as the effective field theory for the two-field inflation in the language of [4] but with additional terms. Note that the above result can be generalized to multi-field inflation as

$$
\Delta \mathcal{L} \propto \sum c_{n_{0}, n_{1}, \ldots, n_{N}}(\vec{T} \cdot \dot{\vec{\delta}})^{n_{0}}\left(\vec{N}_{1} \cdot \dot{\vec{\delta}}\right)^{n_{1}}\left(\vec{N}_{2} \cdot \dot{\vec{\delta}}\right)^{n_{2}} \ldots\left(\vec{N}_{N} \cdot \dot{\vec{\delta}}\right)^{n_{N}}
$$

where $\vec{T}$ and $\vec{N}_{i}$ 's are a set of orthonormal vectors for an $(N+1)$-field model.

\section{CONCLUSIONS}

In this work the effective field theory of multi-field inflation has been studied as a generalization of Weinberg's idea [1] for a single field. In this approach the most general Lagrangian is built by using all the covariant terms of the fields. Though effectively the terms with higher order derivatives are interested in the higher energy scales. In this work we restricted the model to the first correction terms. They results in up to fourth order terms in perturbations. Then due to the physical interests we switched to the adiabatic and entropy formalism. It has been shown that generally these modes can have different speeds of sound. By considering the non-linear terms we studied the non-Gaussinity in this model. It has been shown that the amplitude of non-Gaussianity can be significant when the curvature of the classical path in the phase-space becomes large. For example a sharp turn in the classical path can realize it. However it seems that existence of the higher order derivative terms in the Lagrangian cannot produce large non-Gaussinity. The bottom line for this fact is the strong constraint on the coefficients to keep the effective field theory valid. But there is an idea that it is possible to take the higher order correction terms under control automatically, e.g. by Vainshtein mechanism. This relaxes the constraint on the coefficient of the correction terms and results in large non-Gaussianity. On the other hand the structure of the interacting terms in the Lagrangian predicts the existence of all the shapes of non-Gaussinity with the different amplitude for different cases. But the characteristic feature of the model is that the non-Gaussinities are correlated. That means if there is a local non-Gaussinity due to the entropy mode then certainly there is a non-Gaussinity in adiabatic mode which is equilateral. The amplitude of these different types of non-Gaussinities are not independent.

In contrast to [4], the adiabatic and entropy perturbations are not distinguishable initially. This fact results in the existence of the perturbations as well as their derivatives. In other words the adiabatic and entropy perturbations are not invariant under the shift symmetry of original fields. However a combination of them is invariant under such a symmetry. These combinations are " $\dot{\delta} \sigma-\dot{\theta} \delta s "$ and " $\dot{\delta} s+\dot{\theta} \delta \sigma$ " or in other form $\vec{T} \dot{\vec{\delta}}$ and $\vec{N} . \dot{\vec{\delta}}$ respectively. This result is important for constructing the effective field theory for multi-field inflation and causes the additional terms with respect to what is considered in [4].

\section{Acknowledgments}

We would like to thank B. A. Bassett, H. Firouzjahi, J. Fonseca, H. R. Sepnagi, N. Sivanandam and M. M. SheikhJabbari for their comments. We are grateful of T. Battefeld for his very useful comments and careful reading of the manuscript. We also specially thank P. Creminelli for very fruitful discussions and comments. We would like to thank ICTP for their warm hospitality and support when this work was initiated.

\section{Appendix A: The Most General Lagrangian for Multi-Field Inflation}

In this appendix we will study the most general form of a Lagrangian including up to the fourth order space-time derivatives for multi-field models. It is a straightforward generalization of what has been done in [16] for a single field. To continue let us review the blocks

$$
\varphi, \nabla_{\mu} \varphi, \nabla_{\mu} \nabla_{\nu} \varphi, \nabla_{\mu} \nabla_{\nu} \nabla_{\rho} \varphi, \nabla_{\mu} \nabla_{\nu} \nabla_{\rho} \nabla_{\sigma} \varphi, g_{\mu \nu}, R_{\mu \nu \rho \sigma}, \nabla_{\alpha} R_{\mu \nu \rho \sigma}, \nabla_{\alpha} \nabla_{\beta} R_{\mu \nu \rho \sigma}
$$


which satisfy general covariance. Obviously all the indices can be raised by the metric inverse $g^{\mu \nu}$. To construct the Lagrangian we need to make scalars from the above blocks. The possible terms are listed in [16] and easily can be generalized for more than one field. The most general form of the Lagrangian with the above blocks for a multi field model reads as $\mathcal{L} / \sqrt{g}=$

$$
\begin{aligned}
& \left\{b_{1}^{I J}(\vec{\varphi}) \square \varphi_{I} \square \varphi_{J}+b_{2}^{I J K}(\vec{\varphi}) \nabla_{\mu} \varphi_{I} \nabla^{\mu} \varphi_{J} \square \varphi_{K}+b_{3}^{I J K L}(\vec{\varphi}) \nabla_{\mu} \varphi_{I} \nabla^{\mu} \varphi_{J} \nabla_{\nu} \varphi_{K} \nabla^{\nu} \varphi_{L}+b_{4}^{I J}(\vec{\varphi}) \nabla_{\mu} \varphi_{I} \nabla^{\mu} \varphi_{J}\right. \\
& +b_{5}(\vec{\varphi})+b_{6}^{I J}(\vec{\varphi})\left(\nabla^{\mu} \varphi_{I}\right)\left(\square \nabla_{\mu} \varphi_{J}\right)+b_{7}^{I}(\vec{\varphi})\left(\nabla_{\mu} \nabla_{\nu} \varphi_{I}\right)^{2}+b_{8}^{I J K}(\vec{\varphi})\left(\nabla_{\mu} \varphi_{I}\right)\left(\nabla_{\nu} \varphi_{J}\right)\left(\nabla^{\mu} \nabla^{\nu} \varphi_{K}\right)+b_{9}^{I}(\vec{\varphi}) \nabla^{\mu} \square \nabla_{\mu} \varphi_{I} \\
& +b_{10}^{I}(\vec{\varphi}) \square^{2} \varphi_{I}+b_{11}^{I}(\vec{\varphi})\left(\nabla^{\mu}\right) \nabla_{\mu} \square \varphi_{I}+c_{1}^{I J}(\vec{\varphi}) R \nabla_{\mu} \varphi_{I} \nabla^{\mu} \varphi_{J}+c_{2}^{I J}(\vec{\varphi}) R^{\mu \nu} \nabla_{\mu} \varphi_{I} \nabla_{\nu} \varphi_{J}+c_{3}^{I}(\vec{\varphi}) R \square \varphi_{I} \\
& \left.+c_{4}^{I}(\vec{\varphi})\left(\nabla^{\mu} R\right)\left(\nabla_{\mu} \varphi_{I}\right)+c_{5}(\vec{\varphi}) \square R+c_{6}^{I}(\vec{\varphi}) R_{\mu \nu} \nabla^{\mu} \nabla^{\nu} \varphi_{I}+a_{1}(\vec{\varphi}) R_{\mu \nu \rho \sigma} R^{\mu \nu \rho \sigma}+a_{2}(\vec{\varphi}) R_{\mu \nu} R^{\mu \nu}+a_{3}(\vec{\varphi}) R^{2}+a_{4}(\vec{\varphi}) R\right\}
\end{aligned}
$$

where summation on repeated indices is assumed and $\vec{\varphi}=\varphi_{1}, \varphi_{2}, \ldots, \varphi_{N}$ for $N$ fields. Note that all of the terms in (A1) are not independent up to total derivative terms. In the following we show this fact in details.

$$
\begin{aligned}
& c_{4}^{I}(\vec{\varphi})\left(\nabla^{\mu} R\right)\left(\nabla_{\mu} \varphi_{I}\right)=\nabla^{\mu}\left(c_{4}^{I}(\vec{\varphi}) R \nabla_{\mu} \varphi_{I}\right)-c_{4}^{I}(\vec{\varphi}) R \square \varphi_{I}-R\left(\nabla^{\mu} c_{4}^{I}(\vec{\varphi})\right) \nabla_{\mu} \varphi_{I} \\
& =\nabla^{\mu}\left(c_{4}^{I}(\vec{\varphi}) R \nabla_{\mu} \varphi_{I}\right)-c_{4}^{I}(\vec{\varphi}) R \square \varphi_{I}-R\left(\partial c_{4}^{I}(\vec{\varphi}) / \partial \varphi_{J}\right) \nabla^{\mu} \varphi_{J} \nabla_{\mu} \varphi_{I} \\
& c_{5}(\vec{\varphi}) \square R=\nabla^{\mu}\left(c_{5}(\vec{\varphi}) \nabla_{\mu} R\right)-\left(\nabla^{\mu} c_{5}(\vec{\varphi})\right)\left(\nabla_{\mu} R\right) \\
& =\nabla^{\mu}\left(\left(c_{5}(\vec{\varphi}) \nabla_{\mu} R\right)-\left(\left(\nabla_{\mu} c_{5}(\vec{\varphi})\right) R\right)\right)+\left(\nabla_{\mu} \nabla^{\mu} c_{5}(\vec{\varphi})\right) R \\
& =\nabla^{\mu}\left(\left(c_{5}(\vec{\varphi}) \nabla_{\mu} R\right)-\left(\left(\nabla_{\mu} c_{5}(\vec{\varphi})\right) R\right)\right)+\nabla_{\mu}\left(\left(\partial c_{5}(\vec{\varphi}) / \partial \varphi_{I}\right) \nabla^{\mu} \varphi_{I}\right) R \\
& =\nabla^{\mu}\left(\left(c_{5}(\vec{\varphi}) \nabla_{\mu} R\right)-\left(\left(\nabla_{\mu} c_{5}(\vec{\varphi})\right) R\right)\right)+\left(\frac{\partial^{2} c_{5}(\vec{\varphi})}{\partial \varphi_{I} \partial \varphi_{J}} \nabla_{\mu} \varphi_{I} \nabla^{\mu} \varphi_{J}+\left(\partial c_{5}(\vec{\varphi}) / \partial \varphi_{I}\right) \nabla_{\mu} \nabla^{\mu} \varphi_{I}\right) R \\
& c_{6}^{I}(\vec{\varphi}) R_{\mu \nu}\left(\nabla^{\mu} \nabla^{\nu} \varphi_{I}\right)=\nabla^{\mu}\left(c_{6}^{I}(\vec{\varphi}) R_{\mu \nu} \nabla^{\nu} \varphi_{I}\right)-R_{\mu \nu}\left(\nabla^{\mu} c_{6}^{I}(\vec{\varphi})\right) \nabla^{\nu} \varphi_{I}-c_{6}^{I}(\vec{\varphi})\left(\nabla^{\mu} R_{\mu \nu}\right) \nabla^{\nu} \varphi_{I} \\
& =\nabla^{\mu}\left(c_{6}^{I}(\vec{\varphi}) R_{\mu \nu} \nabla^{\nu} \varphi_{I}\right)-R_{\mu \nu}\left(\partial c_{6}^{I}(\vec{\varphi}) / \partial \varphi_{J}\right) \nabla^{\mu} \varphi_{J} \nabla^{\nu} \varphi_{I}+\frac{1}{2} g_{\mu \nu} c_{6}^{I}(\vec{\varphi})\left(\nabla^{\mu} R\right) \nabla^{\nu} \varphi_{I} \\
& =\nabla^{\mu}\left(c_{6}^{I}(\vec{\varphi}) R_{\mu \nu} \nabla^{\nu} \varphi_{I}\right)-R_{\mu \nu}\left(\partial c_{6}^{I}(\vec{\varphi}) / \partial \varphi_{J}\right) \nabla^{\mu} \varphi_{J} \nabla^{\nu} \varphi_{I}+\frac{1}{2} g_{\mu \nu} \nabla^{\mu}\left(c_{6}^{I}(\vec{\varphi}) R \nabla^{\nu} \varphi_{I}\right) \\
& -\frac{1}{2} g_{\mu \nu} R\left(\nabla^{\mu} c_{6}^{I}(\vec{\varphi})\right) \nabla^{\nu} \varphi_{I}-\frac{1}{2} g_{\mu \nu} c_{6}^{I}(\vec{\varphi}) R \nabla^{\mu} \nabla^{\nu} \varphi_{I} \\
& =\nabla^{\mu}\left(c_{6}^{I}(\vec{\varphi}) R_{\mu \nu} \nabla^{\nu} \varphi_{I}\right)+\frac{1}{2} \nabla^{\mu}\left(c_{6}^{I}(\vec{\varphi}) R \nabla_{\mu} \varphi_{I}\right) \\
& -R_{\mu \nu}\left(\partial c_{6}^{I}(\vec{\varphi}) / \partial \varphi_{J}\right) \nabla^{\mu} \varphi_{J} \nabla^{\nu} \varphi_{I}-\frac{1}{2} R\left(\partial c_{6}^{I}(\vec{\varphi}) / \partial \varphi_{J}\right) \nabla_{\mu} \varphi_{J} \nabla^{\mu} \varphi_{I}-\frac{1}{2} g_{\mu \nu} c_{6}^{I}(\vec{\varphi}) R \nabla^{\mu} \nabla^{\nu} \varphi_{I} \\
& b_{6}^{I J}(\vec{\varphi})\left(\nabla^{\mu} \varphi_{I}\right) \square \nabla_{\mu} \varphi_{J}=b_{6}^{I J}(\vec{\varphi})\left(\nabla^{\mu} \varphi_{I}\right) \nabla^{\nu} \nabla_{\nu} \nabla_{\mu} \varphi_{J}=b_{6}^{I J}(\vec{\varphi})\left(\nabla^{\mu} \varphi_{I}\right) \nabla^{\nu} \nabla_{\mu} \nabla_{\nu} \varphi_{J}+b_{6}^{I J}(\vec{\varphi})\left(\nabla^{\mu} \varphi_{I}\right) \nabla^{\nu}\left[\nabla_{\nu}, \nabla_{\mu}\right] \varphi_{J} \\
& =b_{6}^{I J}(\vec{\varphi})\left(\nabla^{\mu} \varphi_{I}\right) \nabla^{\nu} \nabla_{\mu} \nabla_{\nu} \varphi_{J}+b_{6}^{I J}(\vec{\varphi})\left(\nabla^{\mu} \varphi_{I}\right) \nabla^{\nu}\left[\nabla_{\nu}, \nabla_{\mu}\right] \varphi_{J} \\
& =b_{6}^{I J}(\vec{\varphi})\left(\nabla^{\mu} \varphi_{I}\right) \nabla_{\mu} \square \varphi_{J}+b_{6}^{I J}(\vec{\varphi})\left(\nabla^{\mu} \varphi_{I}\right)\left[\nabla_{\nu}, \nabla_{\mu}\right] \nabla^{\nu} \varphi_{J}+b_{6}^{I J}(\vec{\varphi})\left(\nabla^{\mu} \varphi_{I}\right) \nabla^{\nu}\left[\nabla_{\nu}, \nabla_{\mu}\right] \varphi_{J} \\
& =b_{6}^{I J}(\vec{\varphi})\left(\nabla^{\mu} \varphi_{I}\right) \nabla_{\mu} \square \varphi_{J}+b_{6}^{I J}(\vec{\varphi})\left(\nabla^{\mu} \varphi_{I}\right) R_{\mu \nu} \nabla^{\nu} \varphi_{J}+0 \\
& =\nabla_{\mu}\left(b_{6}^{I J}(\vec{\varphi})\left(\nabla^{\mu} \varphi_{I}\right) \square \varphi_{J}\right)-\left(\nabla_{\mu} b_{6}^{I J}(\vec{\varphi})\right)\left(\nabla^{\mu} \varphi_{I}\right) \square \varphi_{J}-b_{6}^{I J}(\vec{\varphi})\left(\square \varphi_{I}\right)\left(\square \varphi_{J}\right) \\
& +b_{6}^{I J}(\vec{\varphi}) R_{\mu \nu}\left(\nabla^{\mu} \varphi_{I}\right)\left(\nabla^{\nu} \varphi_{J}\right) \\
& =\nabla_{\mu}\left(b_{6}^{I J}(\vec{\varphi})\left(\nabla^{\mu} \varphi_{I}\right) \square \varphi_{J}\right)-\left(\partial b_{6}^{I J}(\vec{\varphi}) / \partial \varphi_{K}\right)\left(\nabla_{\mu} \varphi_{K}\right)\left(\nabla^{\mu} \varphi_{I}\right) \square \varphi_{J} \\
& -b_{6}^{I J}(\vec{\varphi})\left(\square \varphi_{I}\right)\left(\square \varphi_{J}\right)+b_{6}^{I J}(\vec{\varphi}) R_{\mu \nu}\left(\nabla^{\mu} \varphi_{I}\right)\left(\nabla^{\nu} \varphi_{J}\right)
\end{aligned}
$$




$$
\begin{aligned}
& b_{7}^{I J}(\vec{\varphi})\left(\nabla_{\mu} \nabla_{\nu} \varphi_{I}\right)\left(\nabla^{\mu} \nabla^{\nu} \varphi_{J}\right)=\nabla_{\mu}\left(b_{7}^{I J}(\vec{\varphi})\left(\nabla_{\nu} \varphi_{I}\right)\left(\nabla^{\mu} \nabla^{\nu} \varphi_{J}\right)\right)-\left(\nabla_{\mu} b_{7}^{I J}(\vec{\varphi})\right)\left(\nabla_{\nu} \varphi_{I}\right)\left(\nabla^{\mu} \nabla^{\nu} \varphi_{J}\right)-b_{7}^{I J}(\vec{\varphi})\left(\nabla^{\nu} \varphi_{I}\right)\left(\square \nabla_{\nu} \varphi_{J}\right) \\
& =\nabla_{\mu}\left(b_{7}^{I J}(\vec{\varphi})\left(\nabla_{\nu} \varphi_{I}\right)\left(\nabla^{\mu} \nabla^{\nu} \varphi_{J}\right)\right)-\left(\partial b_{7}^{I J}(\vec{\varphi}) / \partial \varphi_{K}\right)\left(\nabla_{\mu} \varphi_{K}\right)\left(\nabla_{\nu} \varphi_{I}\right)\left(\nabla^{\mu} \nabla^{\nu} \varphi_{J}\right) \\
& -b_{7}^{I J}(\vec{\varphi})\left(\nabla^{\nu} \varphi_{I}\right)\left(\square \nabla_{\nu} \varphi_{J}\right) \\
& b_{8}^{I J K}(\vec{\varphi})\left(\nabla_{\nu} \varphi_{I}\right)\left(\nabla_{\mu} \varphi_{J}\right)\left(\nabla^{\nu} \nabla^{\mu} \varphi_{K}\right)=\nabla^{\nu}\left(b_{8}^{I J K}(\vec{\varphi})\left(\nabla_{\nu} \varphi_{I}\right)\left(\nabla_{\mu} \varphi_{J}\right)\left(\nabla^{\mu} \varphi_{K}\right)\right) b_{8}^{I J K}(\vec{\varphi})\left(\square \varphi_{I}\right)\left(\nabla_{\mu} \varphi_{J}\right)\left(\nabla^{\mu} \varphi_{K}\right) \\
& -b_{8}^{I J K}(\vec{\varphi})\left(\nabla_{\nu} \varphi_{I}\right)\left(\nabla^{\nu} \nabla_{\mu} \varphi_{J}\right)\left(\nabla^{\mu} \varphi_{K}\right)-\left(\nabla^{\nu}\left(b_{8}^{I J K}(\vec{\varphi})\right)\left(\nabla_{\nu} \varphi_{I}\right)\left(\nabla_{\mu} \varphi_{J}\right)\left(\nabla^{\mu} \varphi_{K}\right)\right. \\
& =\nabla^{\nu}\left(b_{8}^{I J K}(\vec{\varphi})\left(\nabla_{\nu} \varphi_{I}\right)\left(\nabla_{\mu} \varphi_{J}\right)\left(\nabla^{\mu} \varphi_{K}\right)\right)-b_{8}^{I J K}(\vec{\varphi})\left(\square \varphi_{I}\right)\left(\nabla_{\mu} \varphi_{J}\right)\left(\nabla^{\mu} \varphi_{K}\right) \\
& -b_{8}^{I J K}(\vec{\varphi})\left(\nabla_{\nu} \varphi_{I}\right)\left(\nabla^{\nu} \nabla_{\mu} \varphi_{J}\right)\left(\nabla^{\mu} \varphi_{K}\right)-\left(\partial b_{8}^{I J K}(\vec{\varphi}) / \partial \varphi_{L}\right)\left(\nabla^{\nu} \varphi_{L}\right)\left(\nabla_{\nu} \varphi_{I}\right)\left(\nabla_{\mu} \varphi_{J}\right)\left(\nabla^{\mu} \varphi_{K}\right) \\
& b_{9}^{I}(\vec{\varphi}) \nabla^{\nu} \square \nabla_{\nu} \varphi_{I}=\nabla^{\nu}\left(b_{9}^{I}(\vec{\varphi}) \square \nabla_{\nu} \varphi_{I}\right)-\left(\nabla^{\nu} b_{9}^{I}(\vec{\varphi})\right) \square \nabla_{\nu} \varphi_{I}=\nabla^{\nu}\left(b_{9}^{I}(\vec{\varphi}) \square \nabla_{\nu} \varphi_{I}\right)-\left(\partial b_{9}^{I}(\vec{\varphi}) / \partial \varphi_{J}\right)\left(\nabla^{\nu} \varphi_{J}\right)\left(\square \nabla_{\nu} \varphi_{I}\right) \\
& b_{10}^{I}(\vec{\varphi}) \square^{2} \varphi_{I}=\nabla^{\mu}\left(b_{10}^{I}(\vec{\varphi}) \nabla_{\mu} \square \varphi_{I}\right)-\left(\nabla^{\mu} b_{10}^{I}(\vec{\varphi})\right) \nabla_{\mu} \square \varphi_{I} \\
& =\nabla^{\mu}\left(b_{10}^{I}(\vec{\varphi}) \nabla_{\mu} \square \varphi_{I}\right)-\left(\partial b_{10}^{I}(\vec{\varphi}) / \partial \varphi_{J}\right)\left(\nabla^{\mu} \varphi_{J}\right) \nabla_{\mu} \square \varphi_{I} \\
& =\nabla^{\mu}\left(b_{10}^{I}(\vec{\varphi}) \nabla_{\mu} \square \varphi_{I}\right)-\nabla_{\mu}\left(\left(\partial b_{10}^{I}(\vec{\varphi}) / \partial \varphi_{J}\right)\left(\nabla^{\mu} \varphi_{J}\right) \square \varphi_{I}\right) \\
& +\left(\nabla_{\mu}\left(\partial b_{10}^{I}(\vec{\varphi}) / \partial \varphi_{J}\right)\right)\left(\nabla^{\mu} \varphi_{J}\right) \square \varphi_{I}+\left(\partial b_{10}^{I}(\vec{\varphi}) / \partial \varphi_{J}\right)\left(\square \varphi_{J}\right) \square \varphi_{I} \\
& =\nabla^{\mu}\left(b_{10}^{I}(\vec{\varphi}) \nabla_{\mu} \square \varphi_{I}\right)-\nabla_{\mu}\left(\left(\partial b_{10}^{I}(\vec{\varphi}) / \partial \varphi_{J}\right)\left(\nabla^{\mu} \varphi_{J}\right) \square \varphi_{I}\right) \\
& +\left(\frac{\partial^{2} b_{10}^{I}(\vec{\varphi})}{\partial \varphi_{J} \partial \varphi_{K}}\right)\left(\nabla_{\mu} \varphi_{K}\right)\left(\nabla^{\mu} \varphi_{J}\right) \square \varphi_{I}+\left(\partial b_{10}^{I}(\vec{\varphi}) / \partial \varphi_{J}\right)\left(\square \varphi_{J}\right) \square \varphi_{I} \\
& b_{11}^{I J}(\vec{\varphi})\left(\nabla^{\nu} \varphi_{I}\right)\left(\nabla_{\nu} \square \varphi_{J}\right)=\nabla^{\nu}\left(\varphi_{I} b_{11}^{I J}(\vec{\varphi})\left(\nabla_{\nu} \square \varphi_{J}\right)\right)-\varphi_{I}\left(\nabla^{\mu} b_{11}^{I J}(\vec{\varphi})\right)\left(\nabla_{\nu} \square \varphi_{J}\right)-\varphi_{I} b_{11}^{I J}(\vec{\varphi}) \square^{2} \varphi_{J} \\
& =\nabla^{\nu}\left(\varphi_{I} b_{11}^{I J}(\vec{\varphi})\left(\nabla_{\nu} \square \varphi_{J}\right)\right)-\left(\varphi_{I}\left(\partial b_{11}^{I J}(\vec{\varphi}) / \partial \varphi_{K}\right)\right) \nabla^{\nu} \varphi_{K}\left(\nabla_{\nu} \square \varphi_{J}\right)-\varphi_{I} b_{11}^{I J}(\vec{\varphi}) \square^{2} \varphi_{J}
\end{aligned}
$$

Due to these relations, the most general Lagrangian (A1) reduces to a Lagrangian with redefined coefficients and vanishing $b_{6}, b_{7}, b_{8}, b_{9}, b_{10}, b_{11}, c_{4}, c_{5}$ and $c_{6}$.

In this step let us deduce the equations of motion for the leading terms. To do this let us assume $a_{4}(\vec{\varphi})=-\frac{M_{P}^{2}}{2}$, $b_{4}^{I J}(\vec{\varphi})=-\frac{M^{2}}{2} \delta^{I J}$ and $b_{5}(\vec{\varphi})=-M_{P}^{2} U(\vec{\varphi})$ by redefinition of the fields without any loss of generality. Now the equations of motion for the $\varphi_{I}$ and $g_{\mu \nu}$ are

$$
M^{2} \square \varphi_{I}=M_{P}^{2} \partial U(\vec{\varphi}) / \partial \varphi_{I}, \quad \quad R_{\mu \nu}=-\left(M^{2} / M_{P}^{2}\right) \delta^{I J} \nabla_{\mu} \varphi_{I} \nabla_{\nu} \varphi_{J}-U(\vec{\varphi}) g_{\mu \nu}
$$

Now by plugging the above relations to the remaining terms of the Lagrangian (A1), it is easy to show that

$$
\begin{gathered}
a_{3}(\vec{\varphi}) R^{2}=a_{3}(\vec{\varphi})\left(-R_{\mu \nu \rho \sigma} R^{\mu \nu \rho \sigma}+4 R_{\mu \nu} R^{\mu \nu}\right) \longrightarrow a_{1}(\vec{\varphi}) R_{\mu \nu \rho \sigma} R^{\mu \nu \rho \sigma}+a_{2}(\vec{\varphi}) R_{\mu \nu} R^{\mu \nu} . \\
b_{1}^{I J}(\vec{\varphi}) \square \varphi_{I} \square \varphi_{J}=\frac{M_{P}^{4}}{M^{4}} b_{1}^{I J}(\vec{\varphi}) \frac{\partial U(\vec{\varphi})}{\partial \varphi_{I}} \frac{\partial U(\vec{\varphi})}{\partial \varphi_{J}} \longrightarrow U(\vec{\varphi}) \\
b_{2}^{I J K}(\vec{\varphi}) \nabla_{\mu} \varphi_{I} \nabla^{\mu} \varphi_{J} \square \varphi_{K}=\frac{M_{P}^{2}}{M^{2}}\left(b_{2}^{I J K}(\vec{\varphi}) \frac{\partial U(\vec{\varphi})}{\partial \varphi_{K}}\right) \nabla_{\mu} \varphi_{I} \nabla^{\mu} \varphi_{J} \longrightarrow b_{4}^{I J}(\vec{\varphi}) \nabla_{\mu} \varphi_{I} \nabla^{\mu} \varphi_{J}
\end{gathered}
$$




$$
\begin{gathered}
c_{1}^{I J}(\vec{\varphi}) R \nabla_{\mu} \varphi_{I} \nabla^{\mu} \varphi_{J}=c_{1}^{I J}(\vec{\varphi})\left(-\frac{M^{2}}{M_{P}^{2}} \delta^{K L} \nabla_{\nu} \varphi_{K} \nabla^{\nu} \varphi_{L}-4 U(\vec{\varphi})\right) \nabla_{\mu} \varphi_{I} \nabla^{\mu} \varphi_{J} \\
\longrightarrow b_{3}^{I J K L}(\vec{\varphi}) \nabla_{\mu} \varphi_{I} \nabla^{\mu} \varphi_{J} \nabla_{\nu} \varphi_{K} \nabla^{\nu} \varphi_{L}+b_{4}^{I J}(\vec{\varphi}) \nabla_{\mu} \varphi_{I} \nabla^{\mu} \varphi_{J} \\
c_{2}^{I J}(\vec{\varphi}) R^{\mu \nu} \nabla_{\mu} \varphi_{I} \nabla_{\nu} \varphi_{J}=c_{2}^{I J}(\vec{\varphi})\left(-\frac{M^{2}}{M_{P}^{2}} \delta^{K L} \nabla^{\mu} \varphi_{K} \nabla^{\nu} \varphi_{L}-U(\vec{\varphi}) g^{\mu \nu}\right) \nabla_{\mu} \varphi_{I} \nabla_{\nu} \varphi_{J} \\
\longrightarrow b_{3}^{I J K L}(\vec{\varphi}) \nabla_{\mu} \varphi_{I} \nabla^{\mu} \varphi_{J} \nabla_{\nu} \varphi_{K} \nabla^{\nu} \varphi_{L}+b_{4}^{I J}(\vec{\varphi}) \nabla_{\mu} \varphi_{I} \nabla^{\mu} \varphi_{J} \\
c_{3}^{I}(\vec{\varphi}) R \square \varphi_{I}=\frac{M_{P}^{2}}{M^{2}} c_{3}^{I}(\vec{\varphi}) \frac{\partial U(\vec{\varphi})}{\partial \varphi_{I}} R \longrightarrow a_{4}(\vec{\varphi}) R
\end{gathered}
$$

Due to the above relations and again a redefinition of $\vec{\varphi}, U(\vec{\varphi}), b_{3}^{I J K L}(\vec{\varphi}), a_{1}(\vec{\varphi})$ and $a_{2}(\vec{\varphi})$ the most general form of the Lagrangian becomes

$$
\begin{aligned}
\mathcal{L} & =\sqrt{g}\left\{b_{3}^{I J K L}(\vec{\varphi}) \nabla_{\mu} \varphi_{I} \nabla^{\mu} \varphi_{J} \nabla_{\nu} \varphi_{K} \nabla^{\nu} \varphi_{L}-\frac{M^{2}}{2} \delta^{I J} \nabla_{\mu} \varphi_{I} \nabla^{\mu} \varphi_{J}-M_{P}^{2} U(\vec{\varphi})\right. \\
& \left.+a_{1}(\vec{\varphi}) R_{\mu \nu \rho \sigma} R^{\mu \nu \rho \sigma}+a_{2}(\vec{\varphi}) R_{\mu \nu} R^{\mu \nu}-\frac{M_{P}^{2}}{2} R\right\}
\end{aligned}
$$

which exactly reduces to what is deduced in [1] for a single field model.

\section{Appendix B: Comparison With Gordon et al. [10]}

It is obvious that the LHS of (17) is exactly compatible with equations (47) and (48) in [10]. The RHS of equation (47) in [10] can be written as $2 \dot{\theta} \dot{\delta} s+2\left(\ddot{\theta}-\frac{V_{\sigma}}{\dot{\sigma}} \dot{\theta}\right) \delta s$. So to show that our calculation is compatible with this result one has to show that $\ddot{\theta}+\left(3 H+2 \frac{\ddot{\sigma}}{\dot{\sigma}}\right) \dot{\theta}+V_{\sigma s}=0$. To show the RHS is also the same let us start by pointing out that

$$
\begin{aligned}
& \dot{\theta}=\frac{1}{\dot{\sigma}^{2}}(\dot{\varphi} \ddot{\chi}-\dot{\chi} \ddot{\varphi})=\frac{1}{\dot{\sigma}^{2}}\left(\dot{\chi} V_{\varphi}-\dot{\varphi} V_{\chi}\right) \quad \longrightarrow \quad \ddot{\theta}=-2 \frac{\ddot{\sigma}}{\dot{\sigma}^{3}}\left(\dot{\chi} V_{\varphi}-\dot{\varphi} V_{\chi}\right)+\frac{1}{\dot{\sigma}^{2}}\left(\ddot{\chi} V_{\varphi}-\ddot{\varphi} V_{\chi}\right)-V_{\sigma s} \\
& \longrightarrow \ddot{\theta}+2 \frac{\ddot{\sigma}}{\dot{\sigma}} \dot{\theta}+V_{\sigma s}=\frac{1}{\dot{\sigma}^{2}}\left(\ddot{\chi} V_{\varphi}-\ddot{\varphi} V_{\chi}\right)=3 H \frac{1}{\dot{\sigma}^{2}}\left(\dot{\varphi} V_{\chi}-\dot{\chi} V_{\varphi}\right)=-3 H \dot{\theta}
\end{aligned}
$$

where in above calculation we used Klein-Gordon equations $\ddot{\varphi}+3 H \dot{\varphi}+V_{\varphi}=0, \ddot{\chi}+3 H \dot{\chi}+V_{\chi}=0$ and $\ddot{\sigma}+3 H \dot{\sigma}+V_{\sigma}=0$ due to $\dot{\sigma}^{2}=\dot{\varphi}^{2}+\dot{\chi}^{2}$. To compare the second equation in (17) and (48) in [10] it should be checked that if the coefficients of $\dot{\delta \sigma}$ and $\delta \sigma$ are same or not. The former is trivial and the latter results in checking $2 \dot{\theta} \frac{\ddot{\sigma}}{\dot{\sigma}}=-\left(\ddot{\theta}+3 H \dot{\theta}+V_{\sigma s}\right)$. This equality has been already checked in the above. So the result is exactly compatible with [10].

[1] S. Weinberg, Effective Field Theory for Inflation, Phys. Rev. D77 (2008) 123541, arXiv:0804.4291 [hep-th].

[2] C. Cheung, P. Creminelli, A. L. Fitzpatrick, J. Kaplan and L. Senatore, The Effective Field Theory of Inflation, JHEP 0803 (2008) 014, arXiv:0709.0293 [hep-th].

[3] P. Creminelli, M. A. Luty, A. Nicolis and L. Senatore, Starting the universe: Stable violation of the null energy condition and non-standard cosmologies, JHEP 0612 (2006) 080 arXiv:hep-th/0606090,

N. Bartolo, M. Fasiello, S. Matarrese and A. Riotto, Large non-Gaussianities in the Effective Field Theory Approach to Single-Field In ation: the Bispectrum, JCAP 1008 (2010) 008, arXiv:1004.0893 [astro-ph.CO],

N. Bartolo, M. Fasiello, S. Matarrese and A. Riotto, Large non-Gaussianities in the Effective Field Theory Approach to Single-Field In ation: the Trispectrum, JCAP 1009 (2010) 035, arXiv:1006.5411 [astro-ph.CO].

[4] L. Senatore and M. Zaldarriaga, The Effective Field Theory of Multifield Inflation, arXiv:1009.2093v1 [hep-th].

[5] S. Cespedes, V. Atal and G. A. Palma, On the importance of heavy fields during inflation, arXiv:1201.4848 [hep-th], G. Shiu and J. Xu, Effective Field Theory and Decoupling in Multi-field Inflation: An Illustrative Case Study, arXiv:1108.0981 [hep-th],

F. Bernardeau and J.-P. Uzan, Non-Gaussianity in multi-field inflation, Phys. Rev. D66 (2002) 103506, arXiv:hep-ph/0207295,

S. Renaux-Petel and G. Tasinato, Nonlinear perturbations of cosmological scalar fields with non-standard kinetic terms, JCAP 0901 (2009) 012, arXiv:0810.2405 [hep-th]. 
[6] D. Baumann, The Physics of Inflation: A Course for Graduate Students in Particle Physics and Cosmology.

[7] P. Creminelli, On non-gaussianities in single-field inflation, JCAP 0310 (2003) 003, arXiv:astro-ph/0306122.

[8] D. Baumann and D. Green, A Field Range Bound for General Single-Field Inflation, arXiv:1111.3040][hep-th].

[9] C. M. Peterson and M. Tegmark, Testing Multi-Field Inflation: A Geometric Approach, arXiv:1111.0927 [astro-ph.CO], C. M. Peterson and M. Tegmark, Non-Gaussianity in Two-Field Inflation, Phys. Rev. D84 (2011) 023520, arXiv:1011.6675 [astro-ph.CO],

T. Battefeld and R. Easther, Non-Gaussianities in Multi-field Inflation, JCAP 0703 (2007) 020, arXiv:astro-ph/0610296, D. Battefeld and T. Battefeld, On Non-Gaussianities in Multi-Field Inflation ( $N$ fields): Bi- and Tri-spectra beyond SlowRoll, JCAP 0911 (2009) 010, arXiv:0908.4269 [hep-th],

J. Elliston, D. Mulryne, D. Seery and R. Tavakol, Evolution of non-Gaussianity in multi-scalar field models, Int. J. Mod. Phys. A26 (2011) 3821, arXiv:1107.2270 [astro-ph.CO],

S. Yokoyama, T. Suyama and T. Tanaka, Primordial Non-Gaussianity in Multi-Scalar Inflation, Phys. Rev. D77 (2008) 083511, arXiv:0711.2920 [astro-ph],

A. Achucarro, J.-O. Gong, S. Hardeman, G. A. Palma and S. P. Patil, Features of heavy physics in the CMB power spectrum, JCAP 1101 (2011) 030, arXiv:1010.3693 [hep-ph],

C. T. Byrnes and G. Tasinato, Non-Gaussianity beyond slow roll in multi-field inflation, JCAP 0908 (2009) 016, arXiv:0906.0767 [astro-ph.CO].

[10] C. Gordon, D. Wands, B. A. Bassett and R. Maartens, Adiabatic and entropy perturbations from inflation, Phys. Rev. D63 (2001) 023506, arXiv:astro-ph/0009131

[11] X. Chen, Primordial Non-Gaussianities from Inflation Models, Adv. Astron. (2010) 638979, arXiv:1002.1416 [astro-ph.CO].

[12] A. Achucarro, J.-O. Gong, S. Hardeman, G. A. Palma and S. P. Patil, Effective theories of single field inflation when heavy fields matter, arXiv:1201.6342 [hep-th].

[13] C. M. Peterson and M. Tegmark, Testing Two-Field Inflation, Phys. Rev. D83 (2011) 023522, arXiv:1005.4056 [astroph.CO].

[14] C. Burrage, C. de Rham, D. Seery and A. J. Tolley, Galileon inflation, JCAP 1101 (2011) 014, arXiv:1009.2497 [hep-th].

[15] A. I. Vainshtein, Phys. Lett. B 39 (1972) 393.

[16] E. Elizalde, A. G. Jacksenaev, S. D. Odintsov and I. L. Shapiro, A Four-Dimensional Theory for Quantum Gravity with Conformal and Nonconformal Explicit Solutions, Class. Quant. Grav. 12 (1995)1385-1400, arXiv:hep-th/9412061.

[17] Here we report Weinberg's idea very quickly without any details. But in the following when we are going to study its generalization we will do it in details in Appendix A

[18] Just remember that the $\mathcal{L}$ has dimension of $M^{4}$ and in natural unit $\partial_{\mu}$ has dimension of $M^{-1}$.

[19] Actually it is the second term of an expansion with respect to the inverse of $M^{2}$ i.e. " $M^{2}, 1, M^{-2}, \ldots$ ". The first term is "- $\frac{M^{2}}{2} g^{\mu \nu} \partial_{\mu} \varphi \partial_{\nu} \varphi$ " in (1).

[20] In 1] instead of Riemann and Ricci tensors in (4), Weyl tensor has been used.

[21] To do this one should set $I=J=K=L=1$.

[22] It is obvious if one expands the correction terms in (4) or A1) for more than four derivative terms then the higher order perturbations show themselves.

[23] The potential term in the second order of perturbations should be added to (16) to make our results comparable with [10]. This term is $-\frac{1}{2}\left(V_{\sigma \sigma} \delta \sigma^{2}+V_{\sigma s} \delta \sigma \delta s+V_{s s} \delta s^{2}\right)$.

[24] Here we do not expand $\vec{T} \cdot \dot{\vec{\delta}}$ and $\vec{N} . \dot{\vec{\delta}}$ since they contain no common terms to factorize. So their expansion may cause just messy stuffs without any physical interests.

[25] Note that we employ the standard definition of $c_{s}$. It means we skip the interaction terms between $\delta \sigma$ and $\delta s$ which exist even in quadratic level.

[26] We here just consider the terms containing time derivatives and not any spatial derivatives. However the procedure is same.

[27] Note that the coefficient of $(\vec{T} . \dot{\vec{\delta}})^{2}$ in (18) is 6 . But by comparison to (19), 2 of 6 appear in definition of the speed of sound, $c_{s}$, (exactly same as the second line in (3)) and what remains is $4(\vec{T} \cdot \dot{\vec{\delta}})^{2}$.

[28] Note that due to the first equation of motion in (17) the $\delta s$ sources $\delta \sigma$.

[29] Note that except here in the rest of the paper we assumed that $g_{i}$ 's are constant as a matter of simplification. But here we would like to show how the functionality of $g_{i}$ 's may affect the final result.

[30] Note that the most general form of the potential term can be supposed. But as mentioned in [8], in the slow-roll regime there is no interesting non-Gaussianity prediction for single field models. However for multi-field models the potential term can result in large non-Gaussianity which considered in [9]. Here, we restrict our calculations to kinetic terms.

[31] Note that here we just consider the time derivative since in the discussion of this section there is no difference between our model and [4] for the terms containing spatial derivatives. This is because the background is not spatial dependent. Remember that the angle of rotation just depends on time. 